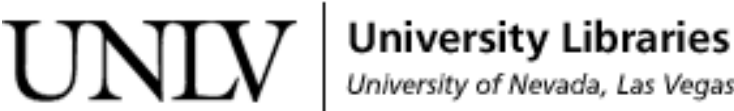

UNLV Theses, Dissertations, Professional Papers, and Capstones

August 2017

\section{Automated Quantification of White Blood Cells in Light Microscopic Images of Injured Skeletal Muscle}

Yang Jiao

University of Nevada, Las Vegas, jiaoy1@unlv.nevada.edu

Follow this and additional works at: https:// digitalscholarship.unlv.edu/thesesdissertations

Part of the Computer Engineering Commons, and the Electrical and Computer Engineering Commons

\section{Repository Citation}

Jiao, Yang, "Automated Quantification of White Blood Cells in Light Microscopic Images of Injured Skeletal Muscle" (2017). UNLV Theses, Dissertations, Professional Papers, and Capstones. 3082.

https://digitalscholarship.unlv.edu/thesesdissertations/3082 


\title{
AUTOMATED QUANTIFICATION OF WHITE BLOOD CELLS IN LIGHT \\ MICROSCOPIC IMAGES OF INJURED SKELETAL MUSCLE
}

\author{
By \\ Yang Jiao \\ Bachelor of Science - Engineering \\ Queen Mary, University of London \\ 2011 \\ Bachelor of Science - Engineering \\ Beijing University of Telecommunications and Posts \\ 2011
}

A thesis submitted in partial fulfillment of the requirements for the

Master of Science - Electrical Engineering

Department of Electrical and Computer Engineering

Howard R. Hughes College of Engineering

The Graduate College

University of Nevada, Las Vegas

August 2017 
Copyright 2017 Yang Jiao

All Rights Reserved 


\section{UNV|GRADUATE COLLEGE}

\section{Thesis Approval}

The Graduate College

The University of Nevada, Las Vegas

July 28, 2017

This thesis prepared by

Yang Jiao

entitled

Automated Quantification of White Blood Cells in Light Microscopic Images of Injured Skeletal Muscle

is approved in partial fulfillment of the requirements for the degree of

Master of Science - Electrical Engineering

Electrical and Computer Engineering

Mei Yang, Ph.D.

Examination Committee Chair

Emma Regentova, Ph.D.

Examination Committee Member

Yingtao Jiang, Ph.D.

Examination Committee Member

Barbara St. Pierre Schneider, Ph.D.

Graduate College Faculty Representative
Kathryn Hausbeck Korgan, Ph.D. Graduate College Interim Dean 


\begin{abstract}
Muscle regeneration process tracking and analysis aim to monitor the injured muscle tissue section over time and analyze the muscle healing procedure. In this procedure, as one of the most diverse cell types observed, white blood cells (WBCs) exhibit dynamic cellular response and undergo multiple protein expression changes. The characteristics, amount, location, and distribution compose the action of cells which may change over time. Their actions and relationships over the whole healing procedure can be analyzed by processing the microscopic images taken at different time points after injury. The previous studies of muscle regeneration usually employ manual approach or basic intensity process to detect and count WBCs. In comparison, computer vision method is more promising in accuracy, processing speed, and labor cost. Besides, it can extract features like cell/cluster size and eccentricity fast and accurately.

In this thesis, we propose an automated quantifying and analysis framework to analyze the WBCs in light microscope images of uninjured and injured skeletal muscles. The proposed framework features a hybrid image segmentation method combining the Localized Iterative Otsu's threshold method assisted by neural networks classifiers and muscle edge detection. In specific, both neural network and convoluted neural network based classifiers are studied and compared. Via this framework, the CD68-positive WBC and 7/4-positive WBC quantification and density distribution results are analyzed for demonstrating the effectiveness of the proposed method.
\end{abstract}




\section{ACKNOWLEDGEMENT}

First and foremost, I would like to thank the chair of my committee, Dr. Mei Yang. She is the one who keeps me focus on my target. Besides, she is such a knowledgeable person that can always provide advice when I encounter difficulties.

I am grateful to Dr. Emma Regentova and Dr Yingtao Jiang, who are the committee members of my thesis. Their professional advice and suggestions are valuable to me.

And, it is my honor to have Dr. Barbara St. Pierre Schneider as the graduate college representative in my committee. She is an expert of biology and human health. And her professional view in these fields contributes significantly in this thesis.

I would like to thank Hananeh Derakhshan for her technical assistance. All experiment images are captured by Hananeh. And she is supported by a UNLV Doctoral Graduate Research Assistantship awarded to Dr. Barbara St. Pierre Schneider.

Finally, this thesis is supported in part by Department of Defense Air Force Grant (FA-7014-10-2-0001) and UNLV Faculty Opportunity Award awarded to Dr. Barbara St. Pierre Schneider. Review of material does not imply Department of the Air Force endorsement of factual accuracy or opinion. 


\section{TABLE OF CONTENTS}

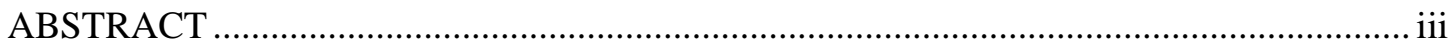

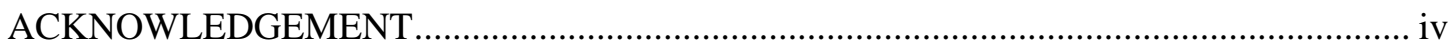

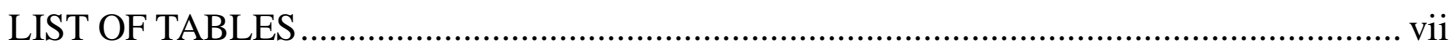

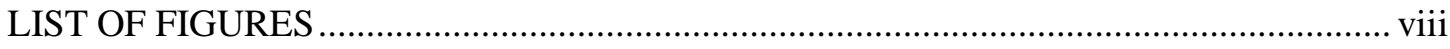

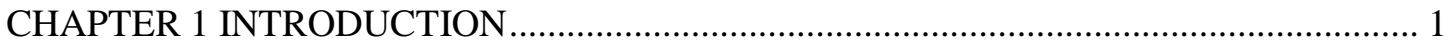

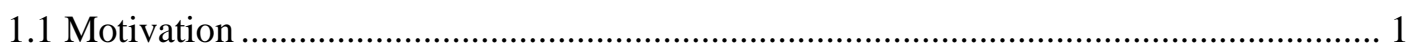

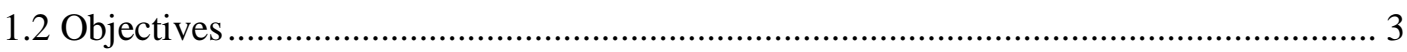

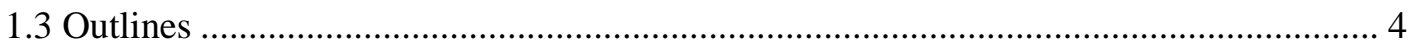

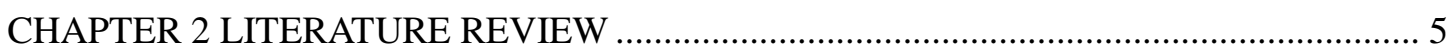

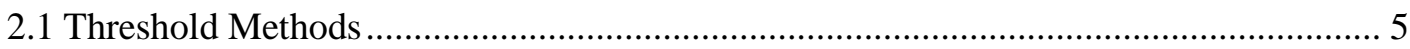

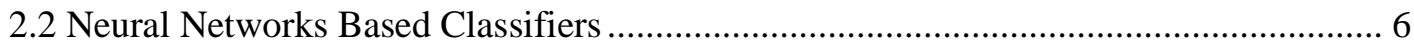

CHAPTER 3 AUTOMATED PROCESSING AND ANALYSIS FRAMEWORK ................. 9

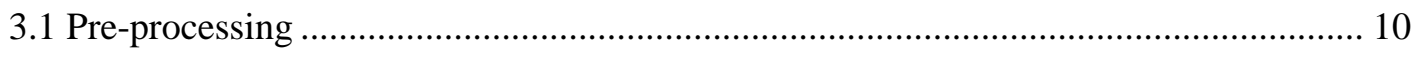

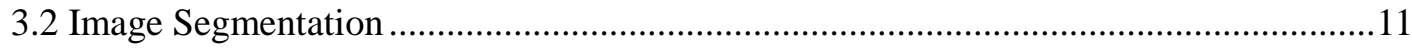

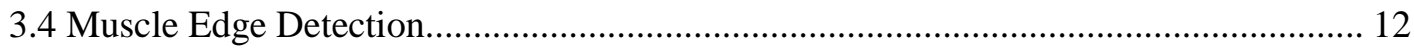

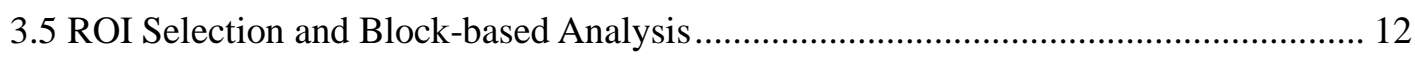

CHAPTER 4 LOCALIZED ITERATIVE OTSU'S THRESHOLD ..................................... 14

4.1 Threshold Value Determination............................................................................... 14

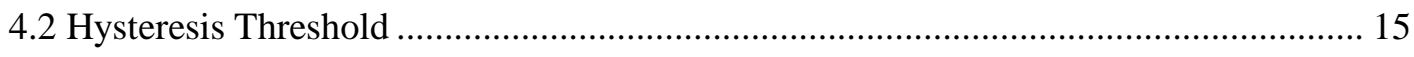

CHAPTER 5 ARTIFICIAL NEURAL NETWORKS WBC DETECTION ........................... 18

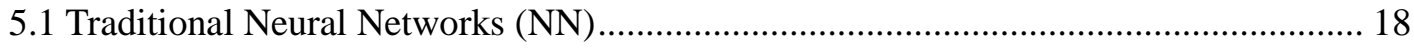

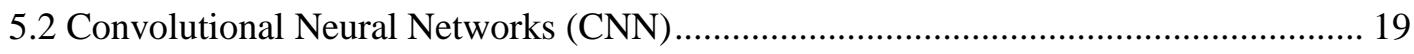

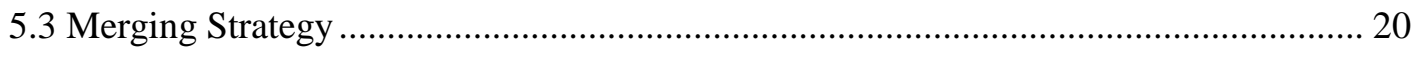

5.4 Training and Testing Result of NN and CNN Classifiers ........................................ 21

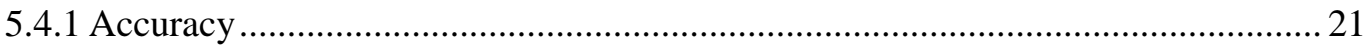

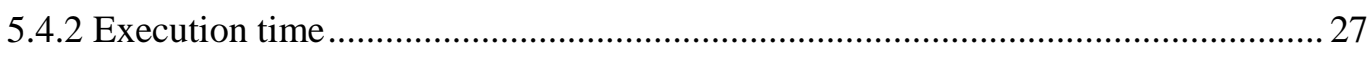

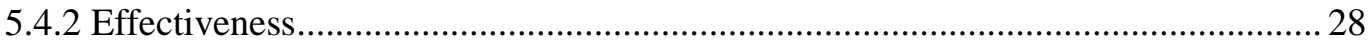

CHAPTER 6 MUSCLE EDGE DETECTION AND ROI SELECTION ……......................... 30

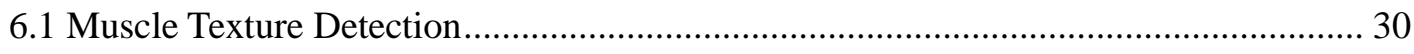

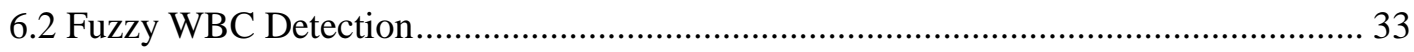




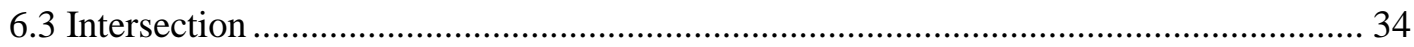

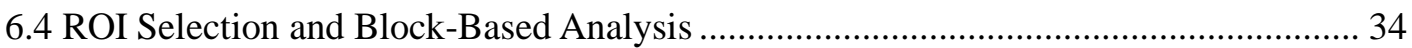

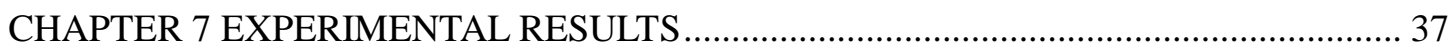

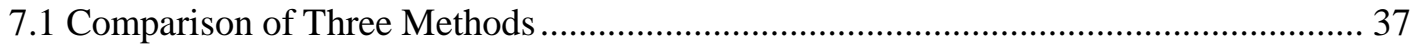

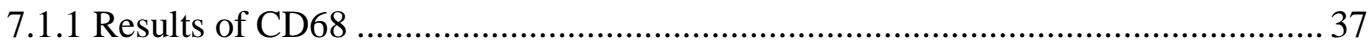

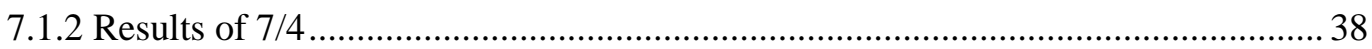

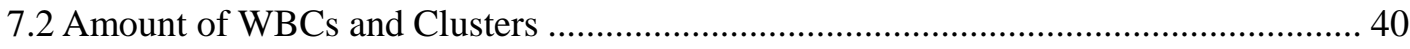

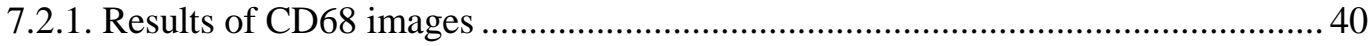

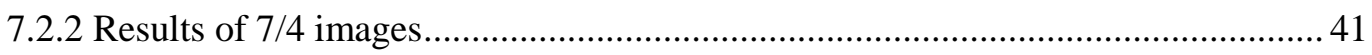

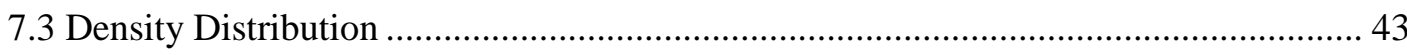

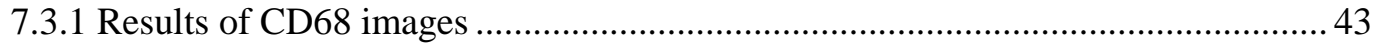

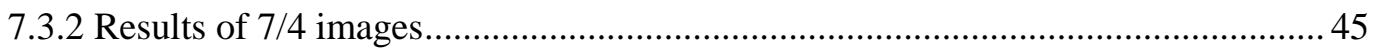

7.4 WBC Model in Muscle Healing Process ............................................................... 47

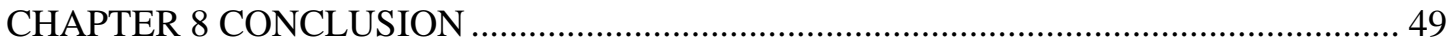

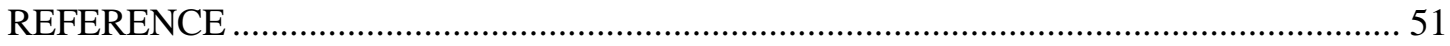

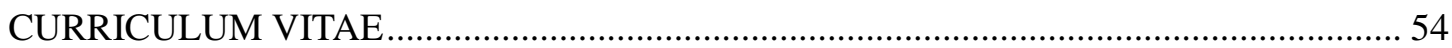




\section{LIST OF TABLES}

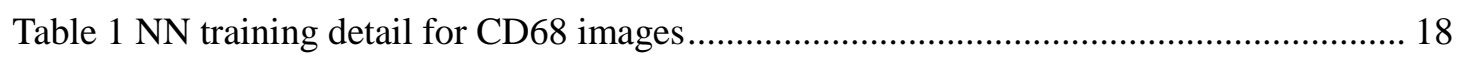

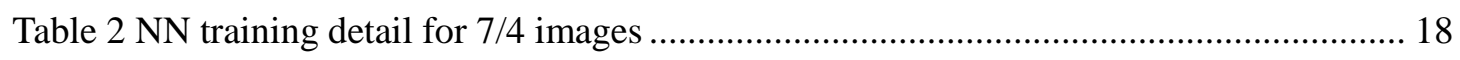

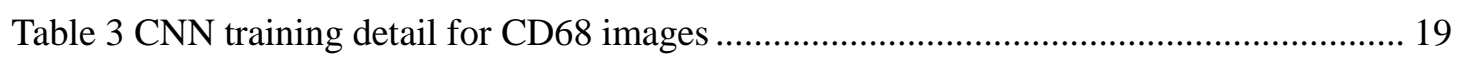

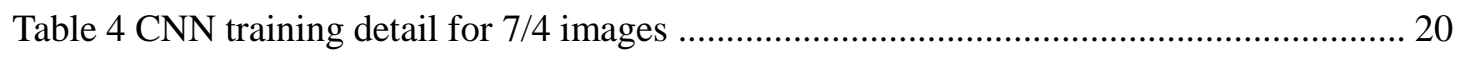

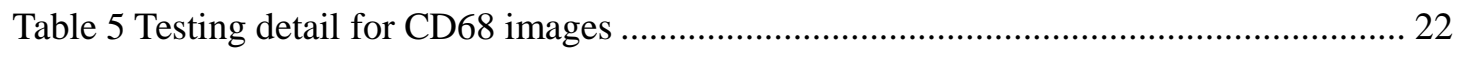

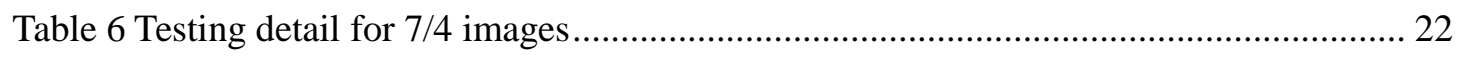

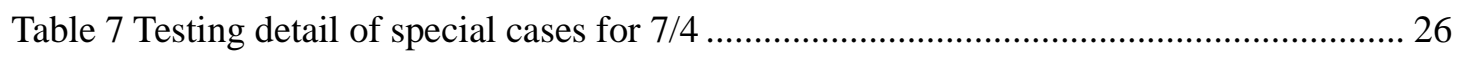

Table 8 Analysis result of CD68-positive WBCs using LIOCNN......................................... 40

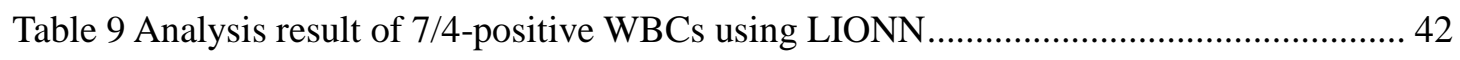




\section{LIST OF FIGURES}

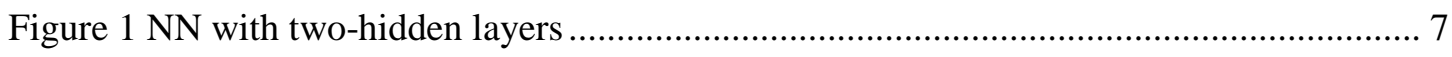

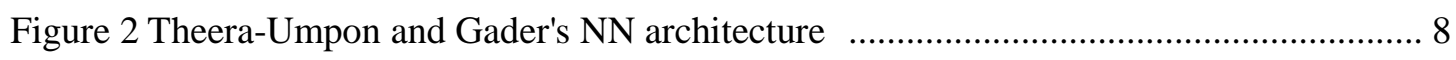

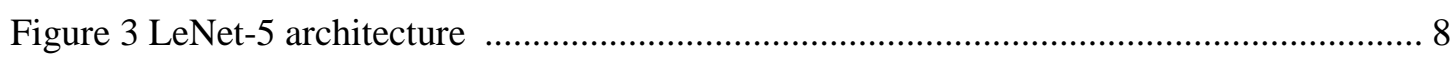

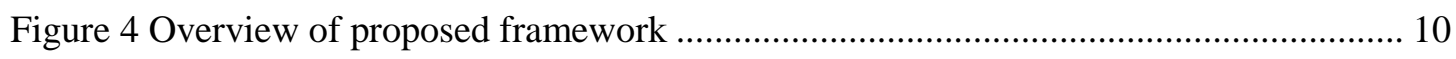

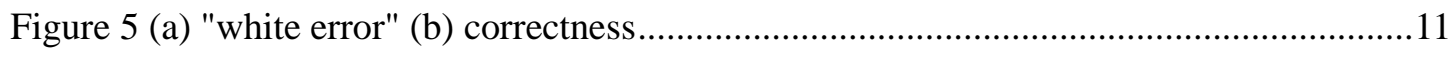

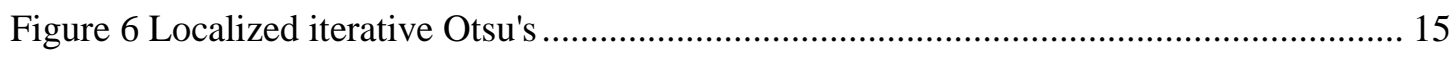

Figure 7 (a) (e) Histogram, (b) (f) Gray scale image, (c) (g) Otsu's results, (d) (h) LI Otsu's

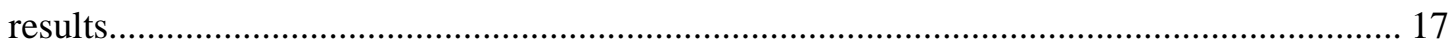

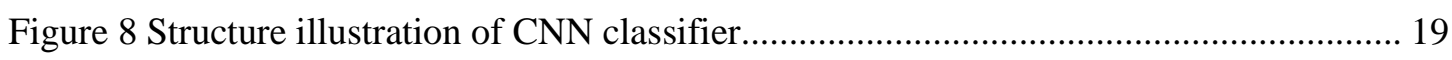

Figure 9 (a) NN testing result for CD68 images (b) CNN testing result for CD68 images (c) NN testing result for $7 / 4$ images (d) CNN testing result for $7 / 4$ images ............................... 22

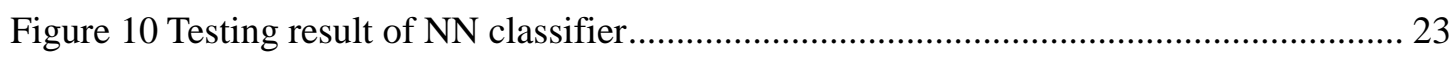

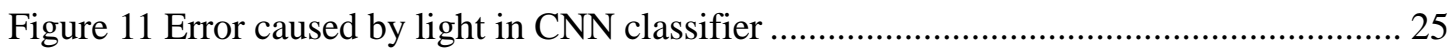

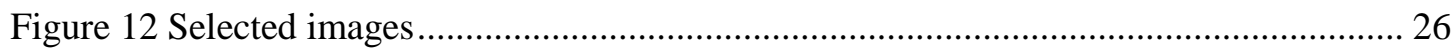

Figure 13 Testing result of $7 / 4$ special case (a) NN (b) CNN ............................................ 27

Figure 14 Training and detection time of classifiers ......................................................... 28

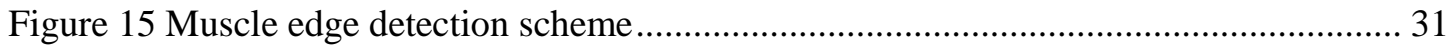

Figure 16 (a) Uninjured muscle image (b) 32-hr after injury muscle image (c) 96-hr after injury muscle image (1) Color image (2) Gray scale image (3) Threshold result (4) Muscle edge detection (5) Canny muscle edge (6) Result of segmentation \& ROI (7) Block histogram

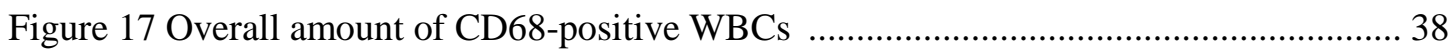

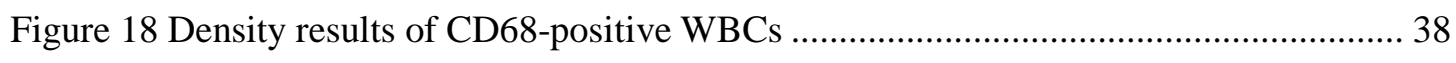

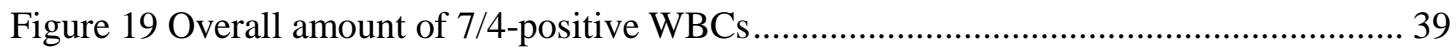

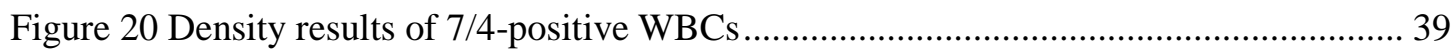

Figure 21 Amount of discrete CD68-positive WBCs .......................................................... 41

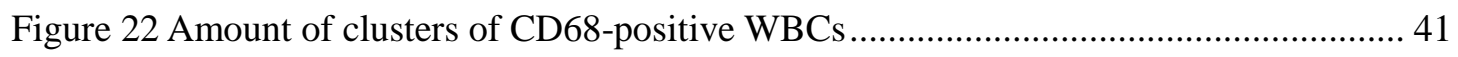

Figure 23 Amount of discrete 7/4-positive WBCs ............................................................... 42

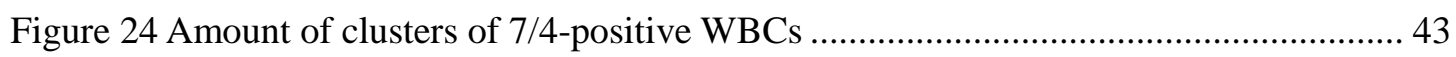

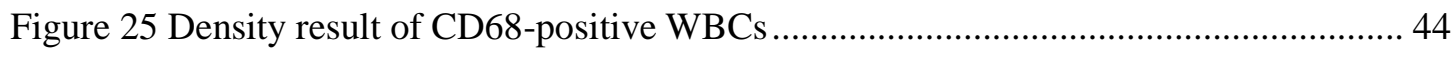




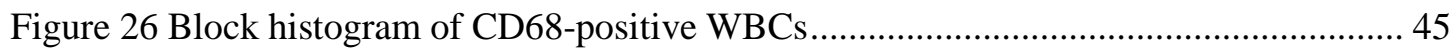

Figure 27 Exponential block histogram of CD68-positive WBCs ......................................... 45

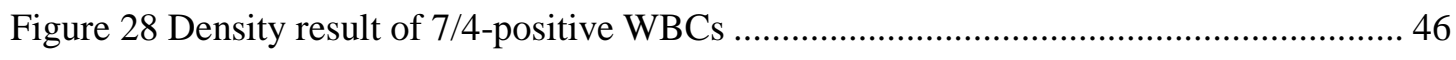

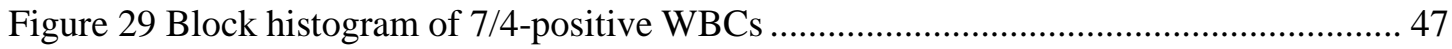

Figure 30 Exponential block histogram of 7/4-positive WBCs............................................ 47 


\section{CHAPTER 1 INTRODUCTION}

Muscle injury commonly occurs during military training. Initial Military Training (IMT)-related musculoskeletal injuries seriously affect U.S. military recruits [19]. Until 2012, approximately $25 \%$ of male and $50 \%$ of female recruits suffered from one or even more muscle injuries during Basic Combat Training (BAT) [38]. These injuries are responsible for more than $80 \%$ disability-related medical discharge in first-year recruits that is estimated to be $\$ 57,500$ per discharged recruit [1][7][21]. Therefore, understanding mechanisms promoting muscle recovery are crucial.

The injured muscle undergoes stages of recovery. Monocytes and macrophages are involved in the inflammatory response process [14]. During this process, WBCs travel from the bone marrow to the injured muscle via the blood vessels. These WBCs exhibit a dynamic response by expressing a variety of proteins (e.g., Gr-1, 7/4, 1A8, CD68, and F4/80) at different post-injury time points [8]. The characteristics of WBCs can be analyzed by processing light microscopic images of uninjured muscles and muscle obtained at different time points after injury (4 to 192 hours). The basic analysis consists of cell recognition and counting.

\subsection{Motivation}

The previous studies of muscle regeneration usually employ manual approach or basic intensity process to detect and count WBCs. Spadaccio et al. [30] and Konstantinovic [13] have employed manual counting approach. In [13], authors used five non-overlapping random selected fields per slide at a magnification of 400x. In [25], ultrasound biomicroscopic (UBM) images are acquired. A 
mean pixel intensity value is calculated and used to represent injury recovery (lower mean values represent higher point of recovery). However, the authors do not quantify cells.

Manual analysis or manually cell counting has defects. First, the success of the manual cell counting depends greatly on the image resolution [28]. At higher resolutions, greater accuracy can be attained; however, this approach is laborious and thus costly. Under high resolution settings, multiple images have to be acquired to cover the whole injured area. Second, manual procedures are also error-prone due to the natural human fatigue after hours of analyses. Evidently, cell characteristics such as cell or cluster size are difficult to obtain manually. Third, it may take long time to process images. Analysis of sophisticated muscle microscope images need carefulness, which may take several rounds to check the results.

On contrary, the computer vision $(\mathrm{CV})$ techniques are promising for analyzing microscopic images, and therefore, are an alternative to the manual methods for biological research. Actually, in 2008, M. Lejeune has achieved semi-automated quantification of diverse subcellular immunohistochemically markers with program Image-Pro Plus [16]. However, it requires researcher to input complex commands at each step. In the literature, image segmentation methods including color space, threshold, and classifiers have been adopted in processing bio-medical images. Most existing work mainly focuses on high magnification image processing and WBC nucleus detection. For images with clustered WBCs and complicated background, a new segmentation method that can adaptively and robustly process and analyze these images is needed. 


\subsection{Objectives}

The main goal of this research is to create a method to automatically process and analyze a large number of light microscopic images of uninjured and injured muscles and extract appearance-based features (number, density, size, shape, and distribution) of WBCs.

$>$ Design of adaptive dynamic threshold

To analyze the actions of different types of proteins, an adaptive dynamic threshold algorithm must be designed to process muscle images. This algorithm must deal with different types of proteins, multiple distribution models, muscle edges, muscle fibers and connective tissues so that satisfactory results can be successfully extracted.

Detection of muscle fibers and connective tissues

Differentiating cells attached with muscle fibers and connective tissues is an important topic in this research. According to their biological features, muscle fibers and connective tissues may be differentiated by distinguishing their edges and shapes. More detail information may be obtained by classifying and studying the different groups of cells.

\section{$>$ Result extraction and analysis}

Valuable results will be extracted and analyzed, including cell and cluster amount, density, cell and cluster size, cell shape, foreground ratio, cell amount that are attached with muscle fibers and connective tissues. Cell-fiber relation changes with time (like location of cells in the periphery of muscle fibers) may be analyzed. These results with aid of biological knowledge will be used to find possible healing equations for different proteins. 


\subsection{Outlines}

The rest of thesis is organized as follows:

Chapter 2 presents the literature review. Our review is focused on existing threshold methods and classifiers used in bio-medical image processing.

Chapter 3 presents the framework of the automated quantification system. This chapter will briefly introduce the composition of the system.

Chapter 4 presents Localized iterative Otsu's threshold (LI Otsu's) method. The LI Otsu's threshold is developed based on Otsu's threshold method. The results obtained by both methods are compared.

Chapter 5 describes the structure, training detail and test results of neural network classifiers. Both traditional neural networks and convolutional neural networks are discussed and evaluated.

Chapter 6 presents the muscle edge detection method and region of interest (ROI) selection rule. This method will help excluding irrelative objects and building density analysis.

Chapter 7 discusses the results of quantification and density analysis of CD68-positive and 7/4-positive WBCs. The trend model is built in this chapter.

Chapter 8 presents the summary and conclusion of this thesis. Besides, we will point out the future work. 


\section{CHAPTER 2 LITERATURE REVIEW}

One of the central problems of computer vision is segmentation of images which is performed for obtaining regions of image related to different objects. The techniques range from color segmentation to intensity threshold, clustering based on either pixel intensities, or secondary (calculated) features fed into trained classifiers. For differentiating WBCs and red blood cells (RBCs), color space processing is typically used. In the papers by Alreza et al., RGB, CMYK, $\mathrm{L}^{*} \mathrm{a} * \mathrm{~b}$ and the gray scale are used to segment nucleus of WBCs [2][34]. Altering color space using different dyeing methods can be used as pre-processing to highlight target object. However, this method is impractical to highlight WBCs with different protein expressions. Next our review is focused on existing threshold methods and neural network based classifiers.

\subsection{Threshold Methods}

In [27], the processing scheme presented by Prinyakupt and Pluempitiwiriyawej employs including morphological processing and histogram processing. Specifically, adjacent objects can be separated by edge detection processing. Among threshold methods, one of the most efficient threshold methods is the Otsu's method [23]. Khobragade et al. demonstrate their method to detect leukemia WBCs. In this paper, histogram processing is employed with traditional Otsu's threshold [15]. Sadeghian et al. use active contour models (snakes) which is based on the gradient vector flow. It is good to describe the contour of object and segment [31]. In [36], HoG and LBP descriptors are applied to train a classifier for detecting WBCs, which achieves $95.56 \%$ accuracy 
An improved Otsu's algorithm that iteratively evaluates the object size and changes threshold is developed in the work by Khan et al. Their method resolves a large object and uneven exposure problems [12]. Ma et al. improves the Otsu's method by drawing a clear boundary between classes of gray values [32]. The work by Sa et al. combines Otsu's algorithm with an edge detector to produce a more accurate segmentation [33]. The 2D Otsu's method is proposed in several papers as an effort to improve robustness of Otsu's method in the presence of noise [26][29]. Besides, applying 2D Otsu's method iteratively can overcome noise and background distortion [9]. 2D Otsu's method has been proved working well for bimodal situation.

The aforementioned work shows the validity of Otsu's method for image segmentation. They mainly focus on high magnification image processing and WBC nucleus detection. Our microscope images are at 100x magnification, in which cell nucleuses are not visible. In addition, complicated background including the muscle edge, connective tissues, and lustered cell steam make WBCs hard to segment and analyze. As such, these methods are not suitable for our work.

\subsection{Neural Networks Based Classifiers}

Other than threshold, neural networks (NNs) can be used to recognize patterns including classifying white blood cells. Bishop describes the function of $\mathrm{NN}$ in his book including singlelayer NNs and two-layer NNs. Unlike single-layer NNs, two layers of weights in NNs are capable of approximating any continuous functional mapping [3]. In NN, the most important component is hidden layer. Each hidden layer contains weight matrix to mix input and generate new output. 


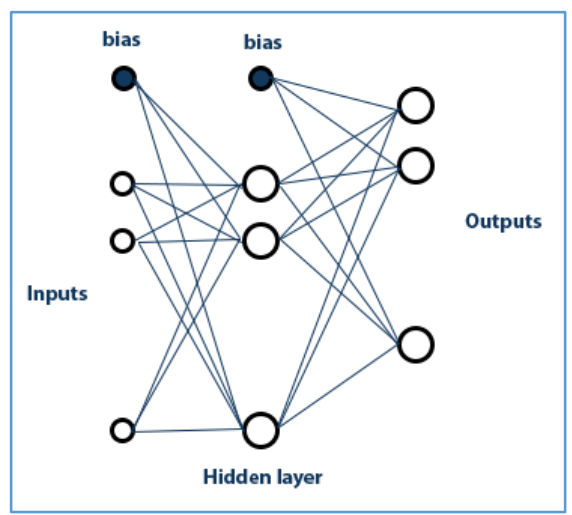

Figure $1 \mathrm{NN}$ with two-hidden layers

In the literature, NNs are used to detect WBCs. Theera-Umpon and Gader present a single hidden layer NN which is used in WBC classification and achieves 78\% accuracy [37]. Particularly, Cellular Neural Networks is presented by Chua and Yang in [5]. Based on Chua's work, Wang presents his work combining color space and morphological image processing with Fuzzy Cellular Neural Networks to achieve WBC detection [35]. The neural network structure in this paper has single hidden layer. Ke shows a novel feature extractor called fuzzy hit/miss operator which is combined with a standard feed-forward network to achieve WBC detection [11]. Fang et al. present particle swarm optimization (PSO) NN structure and realize a remarkable faster training than traditional NN [10]. Manikl et al. extract 7 features from WBC image and utilize MATLAB toolbox to achieve a two-layer feed-forward (single hidden layer) NN to classify 3 types of WBCs [20]. These present work mostly employs single-hidden-layer NNs. Nevertheless, two-hidden-layer NN is proved to have better performance in classification than single-hidden-layer NN [18]. 


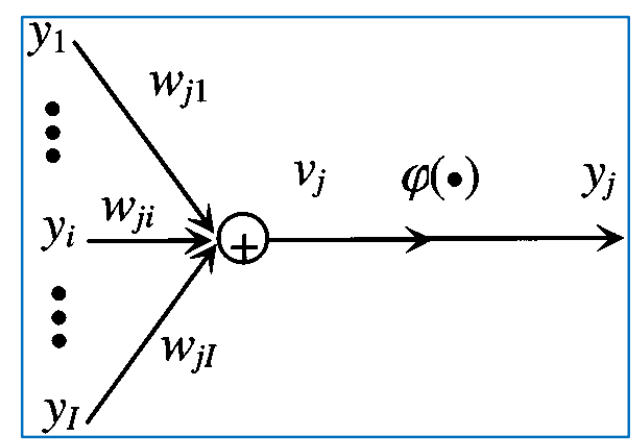

\section{Figure 2 Theera-Umpon and Gader's NN architecture [37]}

In [18], LeCun et al. point out that the traditional fully connected feed-forward neural networks have problems. First, with the increase of parameters and dataset, the training complexity would be challenging. Second, fully connected feed-forward neural networks do not utilize the local features of images. They presented a convolutional neural network (CNN) structure aka. LeNet-5 with 1) local receptive fields; 2) shared weights (or weight replication); and 3) spatial or temporal subsampling has shown better result for multi-class classification. The advantages of CNN are presented: massive sample handling, fast training, spatial feature utilization and better accuracy in ten-class classification [17].

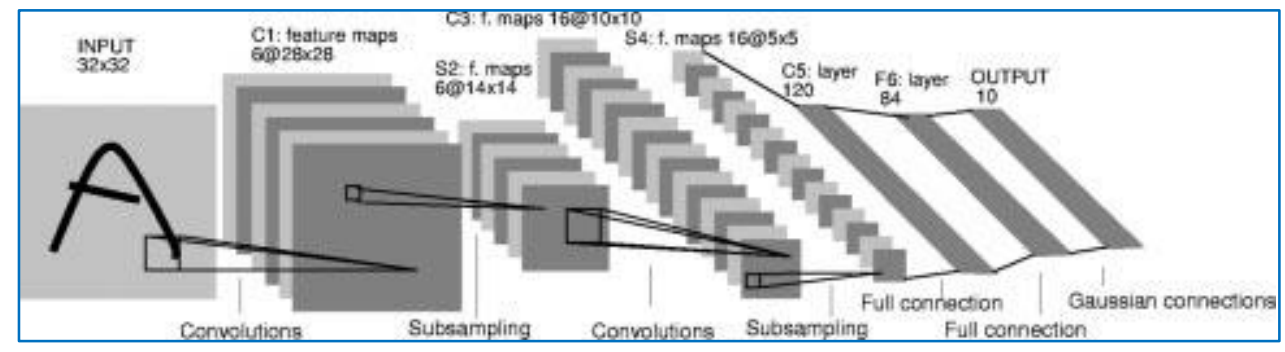

Figure 3 LeNet-5 architecture [17]

$\mathrm{CNN}$ is used in cell differentiation as well. However, this method does not fit this thesis. In this thesis, the CNN classifier will be trained and compared with the NN classifier for WBC detection. 


\section{CHAPTER 3 AUTOMATED PROCESSING AND ANALYSIS FRAMEWORK}

The focus of this work is on segmentation of WBCs and their differentiation from other constituents of muscle images, such as muscle fibers, blood vessels, nerve bundles, and connective tissues. The input microscopic images are of resolution 1600x1200 pixels at 100x magnification. On each slide stained with antibody, WBCs are darker than other constituents; muscle fibers are clearly visible; WBCs can appear as discrete cells or clustered cells. A challenging issue for cell segmentation and quantification is that the cells can be concentrated at the muscle edge. Because the muscle edge can often appear darker than other parts, the edge is excluded from consideration. Also, neural networks can be composed with threshold to correct false detection.

Figure 4 shows the flowchart of the developed framework. It consists of four major steps: pre-processing, image segmentation using the localized iterative Otsu's threshold method assisted by neural network based classifiers, muscle edge detection, and region of interest (ROI) selection and analysis. The objective of each step and methodologies used will be described in the following sections. 


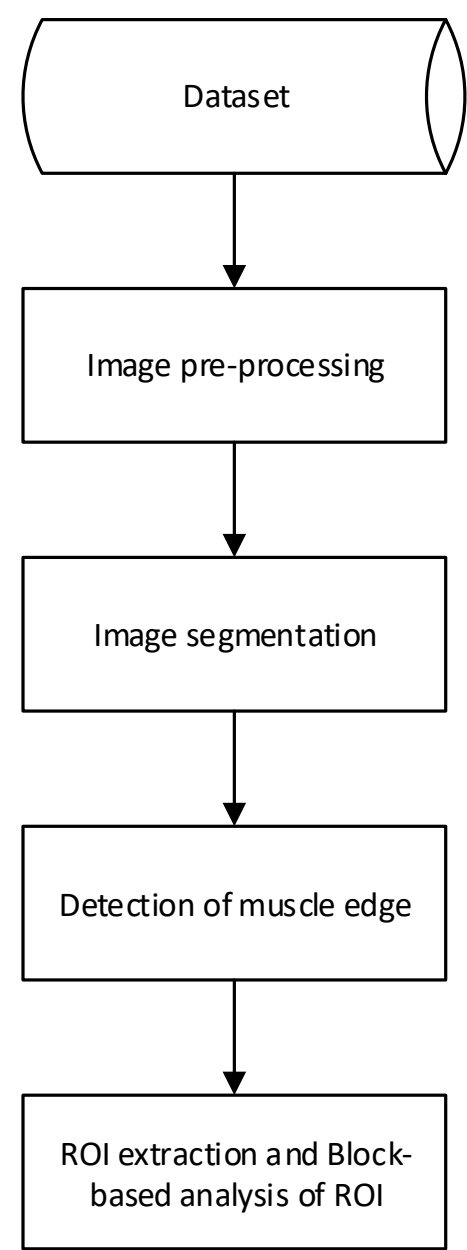

Figure 4 Overview of the proposed framework

\subsection{Pre-processing}

Pre-processing is applied to prepare images for further processing. An input image of 1600x1200

pixels is converted into the gray scale. Then, a global mean intensity value is calculated by Eq. (1)

$$
\mu=\left(\sum_{j}^{J} \sum_{k}^{K} i_{j k}\right) / J K
$$

Where $\mu$ is the mean pixel intensity, $J$ and $K$ represent the width and length of image respectively, and $i$ is the intensity value of pixel at $(j, k)$. 
Image artifacts such as air bubbles are corrected by filling in the pixel values with the calculated mean $\mu$. Figure 5 shows an example of correction of artifacts.

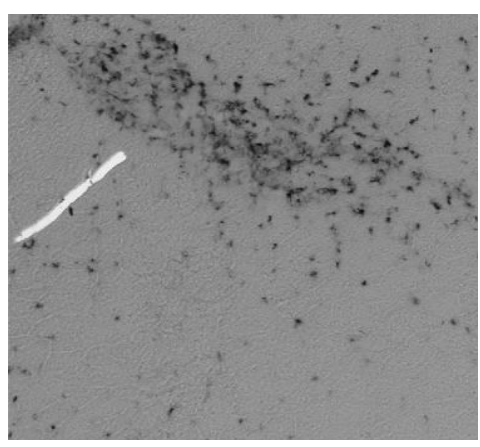

(a)

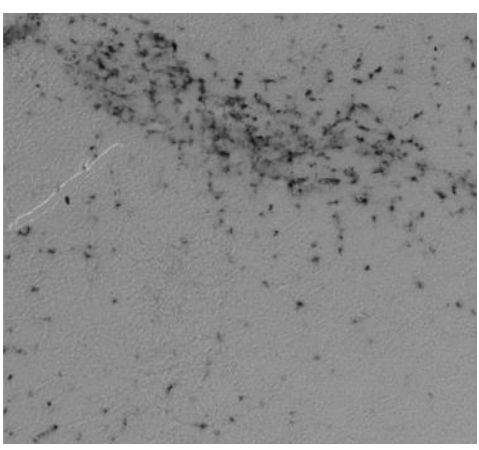

(b)

Figure 5 (a) "white error" (b) correctness

The input image is divided into blocks of 400x400 pixels and the local mean intensity value and the standard deviation $\sigma$ are found by Eq. (1) and Eq. (2).

$$
\sigma=\sqrt{\sum_{j}^{J} \sum_{k}^{K}\left(i_{j k}-\mu_{\text {local }}\right)^{2}}
$$

\subsection{Image Segmentation}

The Localized Iterative Otsu's Threshold (LI Otsu's) Method assisted with neural network based classifiers is proposed to segment WBCs. Intensity variation within and across the images as well as contrast variation are common problems that may be encountered for a given type of miscroscopic images with two-point lighting correction. Since the muscle fibers and the muscle edge can bias segmentation results, a local threshold is calculated. The implementation details of LI Otsu's threshold method will be given in Chapter 4. 
A challenge faced by WBC detection is the fused background caused by staining variation of particular protein, e.g., 7/4. Another challenge can be the staining of connective tissues (CTs). Other than WBCs concentrating on CTs, CTs can be stained as well as the background to cause false positives. As reviewed in Chapter 2, artificial neural networks are used to recognize and classify patterns, which may produce remarkable results. Especially, CNN is boosted with the concept of deep learning and shows a wonderful potential in multiclass classification. To overcome the above challenges, NN and CNN classifiers are adopted to classify WBCs. The results will be merged with those generated from LI Otsu's method to reduce error rate.

\subsection{Muscle Edge Detection}

As discussed before, muscle edge can interfere with the WBC analysis because the edge may falsely acquire a dark color. Muscle edge detection is an important part of the proposed framework. In this step, muscle edge is detected, and WBCs in close proximity to the muscle edge are excluded from subsequent analyses. Chapter 4 describes the muscle edge detection method.

\subsection{ROI Selection and Block-based Analysis}

Via Region of Interest (ROI) selection, areas that are not related to muscle injury are excluded. Typically, ROIs shall not include the muscle edge and the surrounding tissues and the edge of the slide. To resolve the variation caused by uneven exposure, the ROI is divided into blocks of $400 \times 400$ pixels for block-based analysis. Given that an average size of cells is 500 pixels, a discrete cell or a cluster of cells can be differentiated. The following quantities are estimated:

1. Number of discrete WBCs 
2. Number of WBC clusters

3. Average size of a discrete WBC.

4. Total area covered by WBC clusters

5. Number of discrete cells in WBC clusters.

$$
N_{c i c}=\frac{\text { Area }_{\text {cluster }}}{E\left[\text { Size }_{W B C}\right]}
$$

6. Total number of WBCs

7. Number of WBCs per block

8. Frequency of occurrence of a block with certain number of WBCs (histogram). 


\section{CHAPTER 4 LOCALIZED ITERATIVE OTSU'S THRESHOLD}

\subsection{Threshold Value Determination}

For every 400x400 block, a local threshold is obtained by applying the LI Otsu's threshold method, as shown in Figure 6.

Two parameters are pre-set: the expected foreground ratio $F$ and the expected object maximum amount $N$. The method proceeds based on Eq. (4) and Eq. (5).

$$
\sigma_{\omega}^{2}=\omega_{0}(t) \sigma_{0}^{2}(t)+\omega_{1}(t) \sigma_{1}^{2}(t)
$$

where $t$ is threshold, $\omega$ is the probability of class separation by $t, \sigma^{2}$ is the variance of class, $t$ is decided when $\sigma_{\omega}^{2}$ is maximized [23]. If the calculated foreground ratio and the maximum object area do not satisfy the pre-set $F$ and $N$ respectively, one more iteration is needed:

$$
t_{i}=S t_{i-1}
$$

where $i$ is an iteration number, $t$ is a threshold, $S$ is a step size of each iteration. $S$ satisfies $0.8<S<$ 1. For the $i^{\text {th }}$ iteration, $t_{i}$ satisfies Eq. (6)

$$
\frac{\text { Area }_{0}\left(t_{i}\right)}{\text { Area }_{1}\left(t_{i}\right)}<F \text { AND } N\left(t_{i}\right)<N
$$

$t_{i}$ is the determined threshold value. 


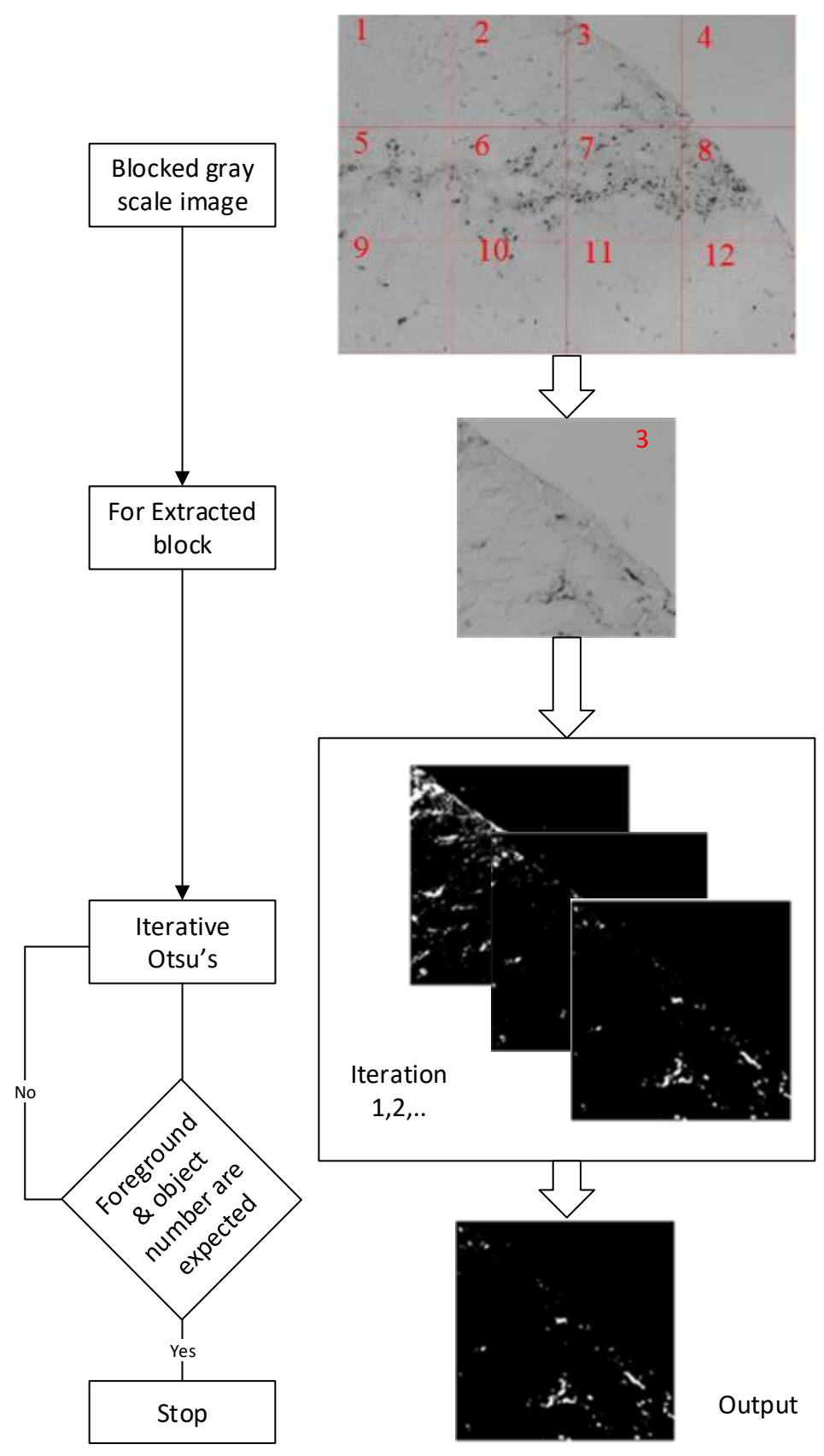

Figure 6 Localized iterative Otsu's

\subsection{Hysteresis Threshold}

The well-known hysteresis threshold is widely used in edge detection. In this thesis, it is used into WBC segmentation. 
After the higher threshold value $t_{i}$ is determined and preliminary objects are segmented, the lower threshold value $t_{i}{ }^{\prime}$ is calculated by Eq. (7)

$$
t_{i}^{\prime}=S^{\prime} t_{i}
$$

Where $S^{\prime}$ is the ratio value.

This method is useful in processing images with complicated background. When a specific threshold value between foreground and complicated background is hard to be found, hysteresis threshold can help to determine the lowest intensity part of a WBC first and then expand to the whole cell.

Figure 7 illustrates the effectiveness of LI Otsu's method using an example image with the muscle edge. Figure 7(a) shows the intensity value histograms of an image whose gray scale image variant is shown in Figure 7(b). The LI Otsu's method obtains the threshold value as 118 producing the segmentation result in Figure 7(d). To compare, the Otsu's method yields the threshold value 146 that gives the segmentation result shown in Figure 7(c). It can be observed that unlike its counterpart the LI Otsu's method retains only WBCs.

Figure 7(e) shows a highly peaked histogram of another image. The Otsu's method fails in recognizing the foreground and the background. It produces the result in Figure $7(\mathrm{~g})$ which mainly contains muscle fibers. Again, the LI Otsu's method manages to treat fibers as a background and present WBCs. 


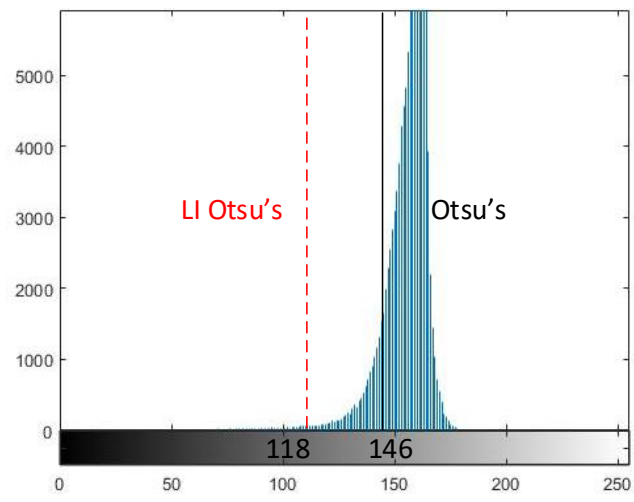

(a)

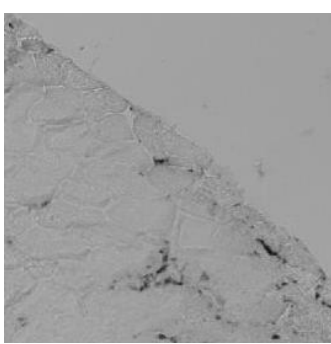

(b)

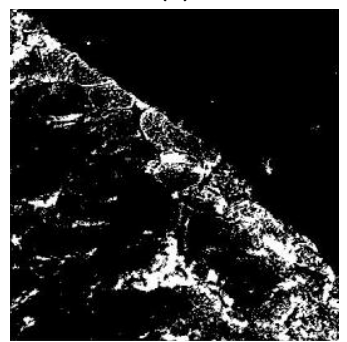

(c)

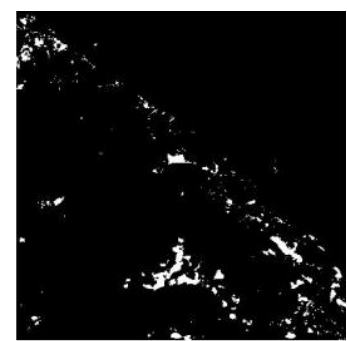

(d)

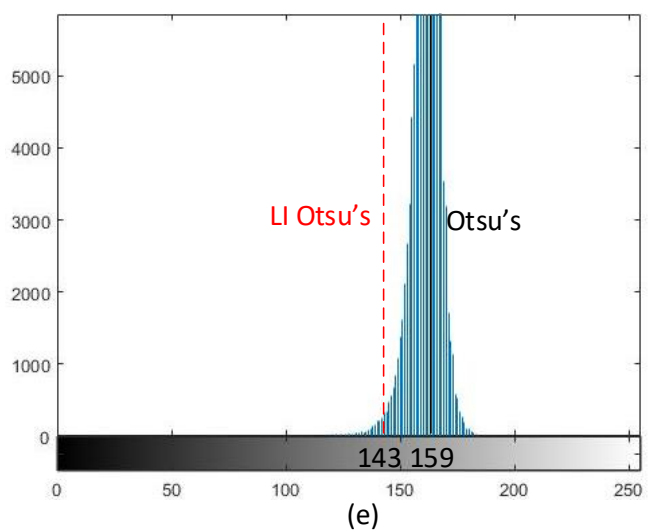

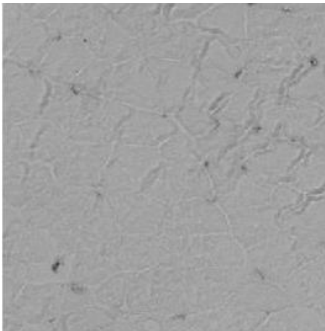

(f)

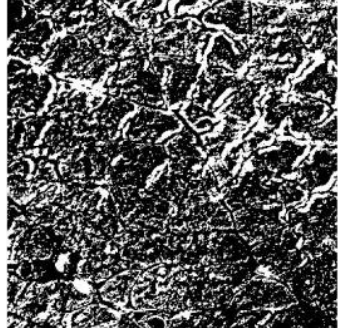

(g)

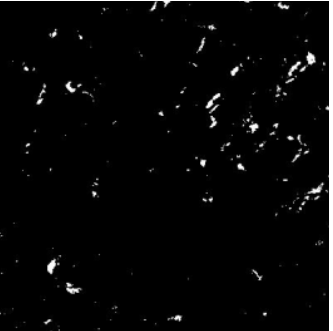

(h)

Figure 7 (a) (e) Histogram, (b) (f) Gray scale image, (c) (g) Otsu's results, (d) (h) LI Otsu's results 


\section{CHAPTER 5 ARTIFICIAL NEURAL NETWORKS WBC DETECTION}

Although CNN is proved as being more accurate on multiclass classification, for classification of two classes, it is not necessary that CNN must have better result than NN. Therefore, both NN and CNN classifiers are trained and tested in this thesis.

\subsection{Traditional Neural Networks (NN)}

As described in [18], a better classification result can be obtained from two-hidden-layer NN than just one hidden layer. Therefore, a two-hidden-layer Feed-forward NN is structured and trained following the method in [6]. The training detail is listed in Table 1 and 2.

Table 1 NN training detail for CD68 images

\begin{tabular}{|l|l|}
\hline \multicolumn{2}{|c|}{ CD68 } \\
\hline Sample selection & From two sample images \\
\hline Class & 2 \\
\hline Positive sample & 1700 \\
\hline Negative sample & 1700 \\
\hline NN layers (hidden layers) & $3(2)$ \\
\hline Hidden layer 1 neurons & 400 \\
\hline Hidden layer 2 neurons & 100 \\
\hline
\end{tabular}

Table 2 NN training detail for 7/4 images

\begin{tabular}{|l|l|}
\hline \multicolumn{2}{|l|}{$7 / 4$} \\
\hline Sample selection & From two sample images \\
\hline Class & 2 \\
\hline Positive sample & 1800 \\
\hline Negative sample & 2000 \\
\hline NN layers (hidden layers) & $3(2)$ \\
\hline Hidden layer 1 neurons & 400 \\
\hline Hidden layer 2 neurons & 100 \\
\hline
\end{tabular}


The number of neurons is determined by experiments. Especially, back-propagation is used on the whole multilayer network. This process is referred to as fine tuning by retraining it on the training data in a supervised fashion.

\subsection{Convolutional Neural Networks (CNN)}

$\mathrm{CNN}$ is usually constructed by seven types of layers. 1) Input layer: used to organize input image data into a 4-D matrix; 2) Convolution layer: used to extract Eigen features; 3) Pooling layer: used to subsample features. The summary statistics are much lower in dimension; 4) ReLU layer: performs a threshold operation, where any input value less than zero is set to zero [22]; 5) Fully-connected layer: multiplies the input by a weight matrix and then adds a bias vector; 6) Softmax layer: The softmax function is also known as the normalized exponential and can be considered as the multi-class generalization of the logistic sigmoid function [3]; 7) Classification layer: the output layer. Based on [24], a 15-layer CNN is established and used in training. Its structure is shown in Figure 8.
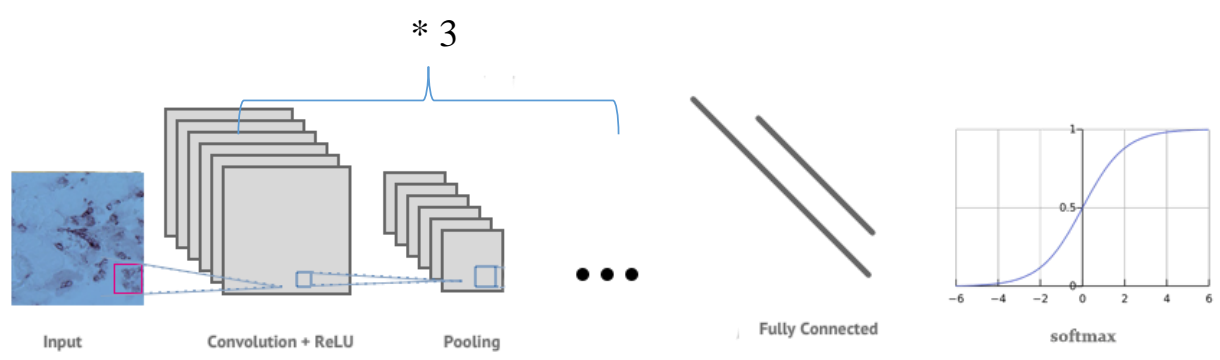

Fulty Connected
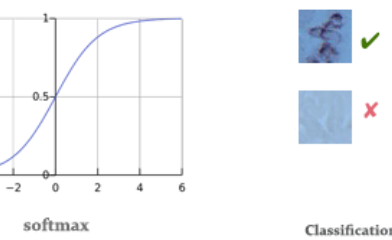

Figure 8 Structure illustration of CNN classifier

The training details of CD68 and 7/4 images are listed in Table 3 and 4, respectively.

Table 3 CNN training detail for CD68 images 


\begin{tabular}{|l|l|}
\hline \multicolumn{2}{|c|}{ CD68 } \\
\hline Sample selection & From two sample images \\
\hline Class & 2 \\
\hline Positive sample & 1700 \\
\hline Negative sample & 1700 \\
\hline CNN layers & 15 \\
\hline
\end{tabular}

Table 4 CNN training detail for 7/4 images

\begin{tabular}{|l|l|}
\hline & $7 / 4$ \\
\hline Sample selection & From two sample images \\
\hline Class & 2 \\
\hline Positive sample & 1800 \\
\hline Negative sample & 2000 \\
\hline CNN layers & 15 \\
\hline
\end{tabular}

\subsection{Merging Strategy}

After obtaining the preliminary detection result from LI Otsu's and neural networks, two results can be merged together to reduce error detection rate. We name the LI Otsu's method assisted by the NN classifier as LIONN, and the Li Otsu's method assisted by the CNN classifier as LIOCNN.

There are two situations of error detection, one is false positive and another is false negative.

Therefore, there are two types of strategies.

a. False negative correction

LI Otsu's algorithm may have false negative results when some special WBCs have too high intensity values than other well dyed WBCs in the same block. However, the NN detection result is beyond block restriction and based on Eigen features. Thus, the NN classifier may have a better chance to detect these special WBCs. In this situation, the following algorithm is applied to adjust the final result. 


\section{Algorithm for False Negative Correction}

Function: Adjusting the detection results when LI Otsu's does not detect an object while NN detects an object in the same location

If $\mathrm{NN}$ detection size $>25 \& \& \mathrm{NN}$ detection size $<1500$

If $f(x, y)<$ level $* \sin (\operatorname{std} * \pi / 20)+0.2$ //Original threshold value multiplied //by a sinusoid to be elastic

$f(x, y)$ can be seen as an object.

\section{Endif}

Endif

b. False positive correction

LI Otsu's algorithm can have false positive results when the background or muscle fiber is false dyed and has similar intensity values with the foreground. However, via learning Eigen features, the $\mathrm{NN}$ detector may be able to distinguish them.

\section{Algorithm for False Positive Correction}

Function: Adjusting the detection results when LI Otsu's detects an object while NN does not detect an object in the same location

If Li Otsu's detection eccentricity > $0.95 / /$ To exclude elongated noises

$f(x, y)$ cannot be seen as an object.

\section{Endif}

If Li Otsu's detection size $>400 / /$ To exclude large background noises $f(x, y)$ cannot be seen as an object.

\section{Endif}

If LI Otsu's detection Eccentricity > $0.97 \& \&$ LI Otsu's detection size > 25,000//To exclude elongated connective tissues

$f(x, y)$ cannot be seen as an object.

Endif

\subsection{Training and Testing Result of NN and CNN Classifiers}

\subsubsection{Accuracy}

The testing details of both NN and CNN classifiers for CD68 and 7/4 are shown in Table 5 and 6, respectively. 
Table 5 Testing detail for CD68 images

\begin{tabular}{|l|l|}
\hline & CD68 \\
\hline Sample selection & From five sample images \\
\hline Class & 2 \\
\hline Positive sample & 120 \\
\hline Negative sample & 120 \\
\hline
\end{tabular}

Table 6 Testing detail for $7 / 4$ images

\begin{tabular}{|l|l|}
\hline & $7 / 4$ \\
\hline Sample selection & From nine sample images \\
\hline Class & 2 \\
\hline Positive sample & 200 \\
\hline Negative sample & 200 \\
\hline
\end{tabular}

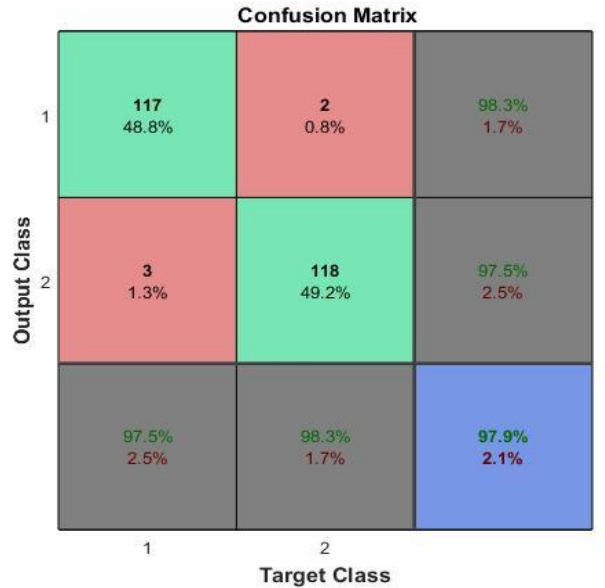

(a)

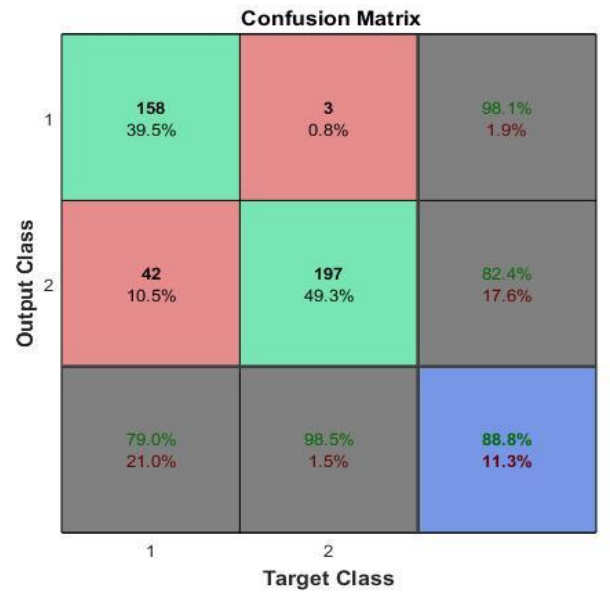

(c)

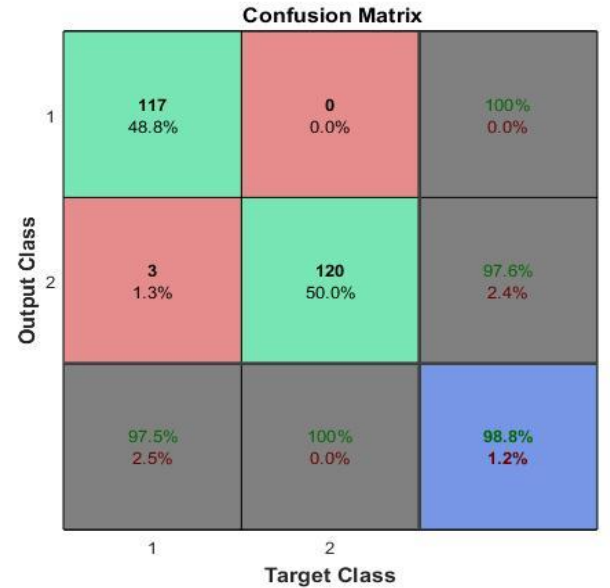

(b)

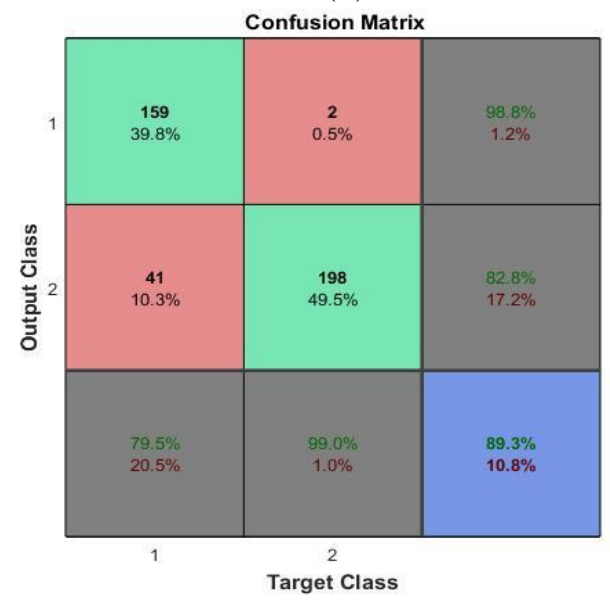

(d)

Figure 9 (a) NN testing result for CD68 images (b) CNN testing result for CD68 images (c) $\mathrm{NN}$ testing result for $7 / 4$ images (d) $\mathrm{CNN}$ testing result for $7 / 4$ images 
The testing result is shown in Figure 9. From the confusion matrix one can see that, if the output result matches the real class, it belongs to the green area, which represents a correct classification. Otherwise, the red area means that either the output result mismatches the actual class. The blue area summarizes the total accuracy and error rate. For example in Figure 9 (a), in class 1, 117 (48.7\%) samples' classification results match their real class, which means correct classification. And $3(1.3 \%)$ class 1 samples are classified as class 2, which means false negative. Therefore, this class has error rate $2.5 \%$. Then, $2(0.8 \%)$ samples which belong to class 2 are misclassified as class 1. For this class, the error rate is $1.7 \%$. For this NN classifier, the total error rate is $2.1 \%$.

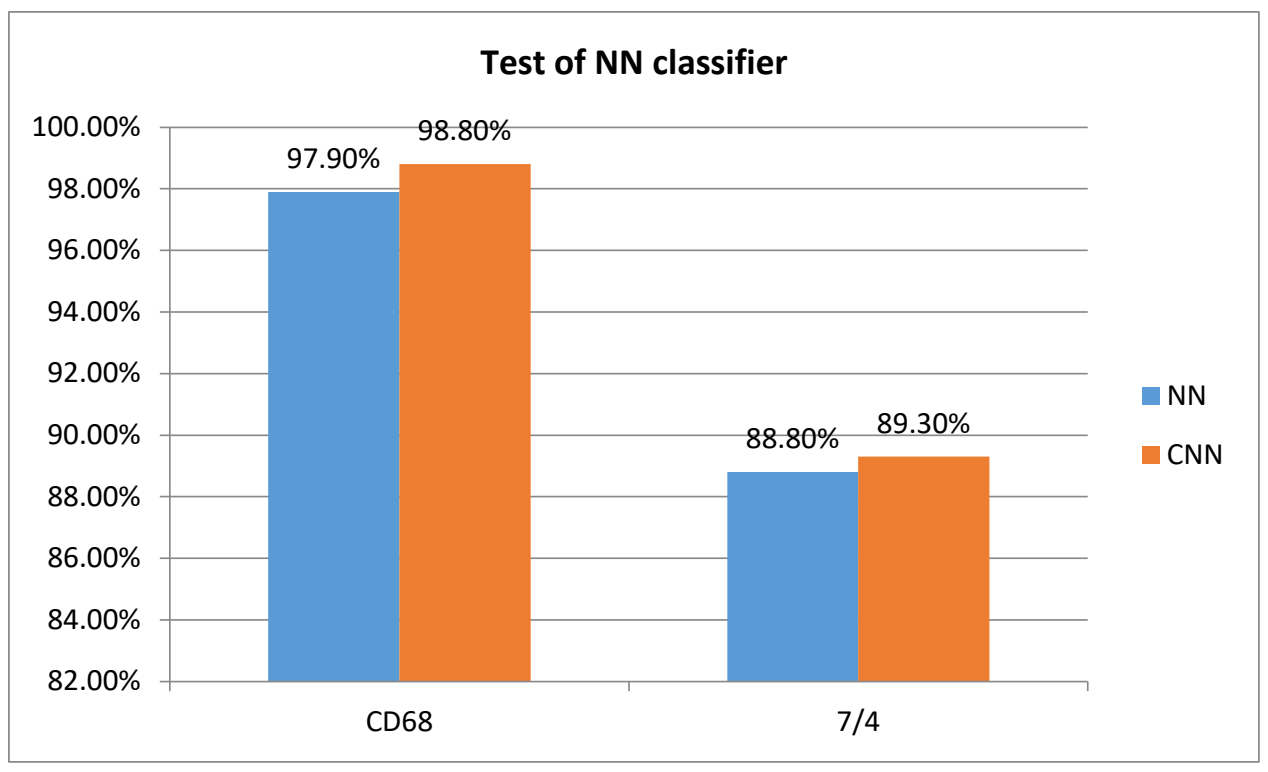

Figure 10 Testing result of NN classifier

From Figure 10, it can be seen that compared with NN classifiers, CNN classifiers have $0.9 \%$ higher accuracy of WBC detection in CD68 images and 0.5\% higher accuracy of WBC detection in 7/4 images.

For CD68 images, the classifiers can generate an up to $98.80 \%$ accuracy result. But for 7/4 images, 
the classifier can generate an $89.30 \%$ accuracy result, which is not as good as that of CD68. The reasons may be attributed to the following aspects.

\section{$>$ Background}

CD68 images have consistent background. Its background includes connective tissues and muscle fibers, but it does not have shading problem (shading: background is artificially dyed by antibody). In 7/4 images, the background can have shading problem. Although the shading area does not have same intensity as WBCs, it may cause problem of inconsistent background and bias classifiers.

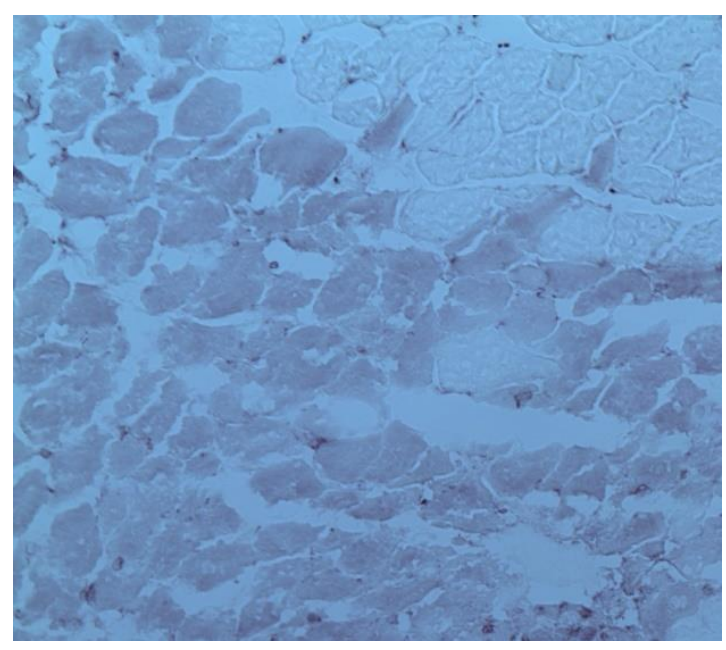

Figure 11 Shading background

$>$ WBCs'shape

In CD68 images, WBCs have consistent shape. They are normally well-dyed roundish or can cluster into a cloud or long string. However, 7/4 images are more complicated. The dyeing intensity and shapes of WBCs vary in 7/4 images, which may complicate feature entry. 
The light of CD68 and 7/4 images uses two-point lighting correction but not five-point correction, which leads to inconsistent intensity within one image. Therefore, intensity and contrast not only vary across images, but also vary within one image. In Figure 11, (a) and (b) show the intensity difference between images and (c) and (d) show that the CNN classifier is sensitive to intensity variation.

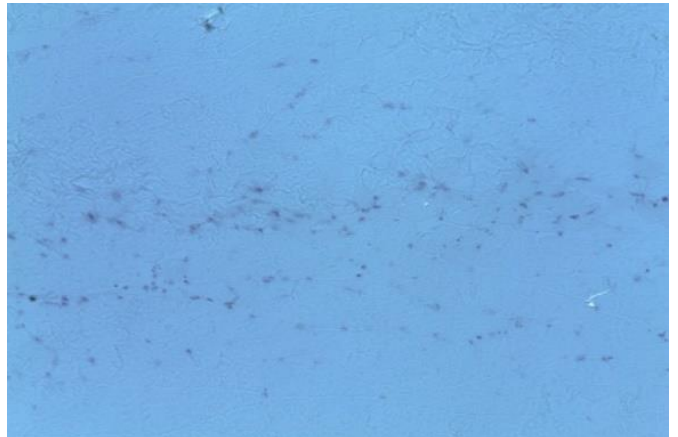

(a)

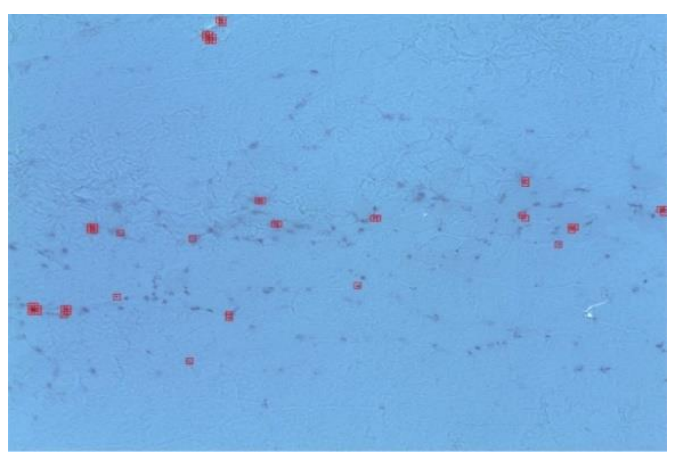

(c)

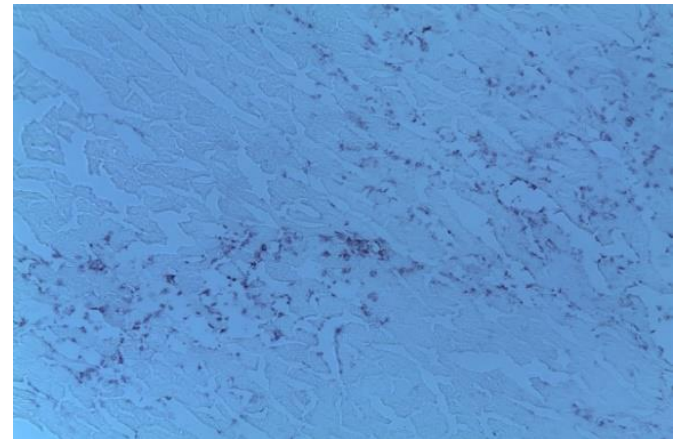

(b)

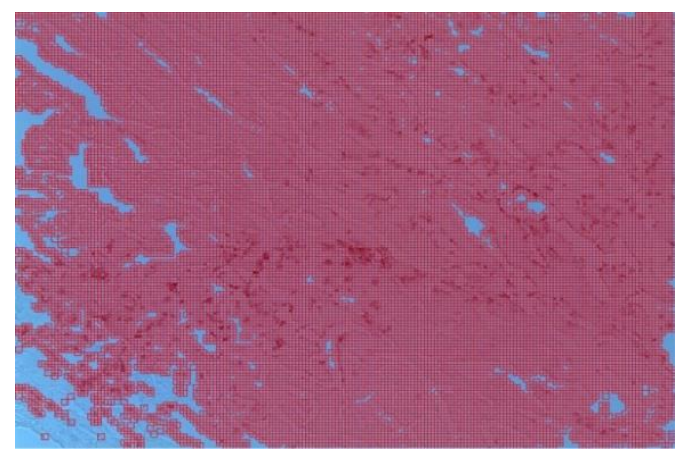

(d)

Figure 12 Error caused by intensity variation in CNN classifier

Due to the above reasons, classifiers of 7/4 images have lower accuracy than that of CD68 images.

On the other hand, the result of $7 / 4$ images using threshold method is not satisfying. Therefore, it is desirable to merge the results of threshold method with that of the classifiers. 
One point need be addressed here. Although CNN tends to have a higher overall accuracy rate, for those inconsistent 7/4 images, the NN classifier can have better detection accuracy than the CNN classifier. For this special case, 40 positive samples and 40 negative samples are selected from a 4-hr image and an 8-hr image. These images are chosen since the WBC' shape can represent these special cases. As the matter of fact, these inconsistent cases occur more frequently at early time points (4-hr, 8-hr, and 24-hr) than at later time points (32-hr, 48-hr, 96-hr, and 192-hr).

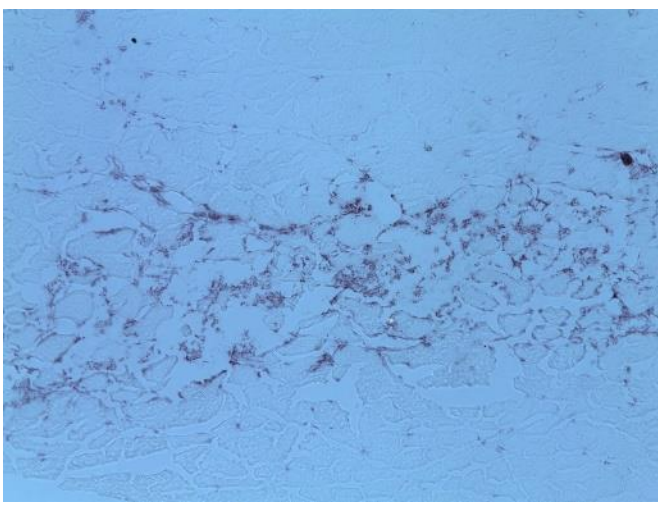

(a)

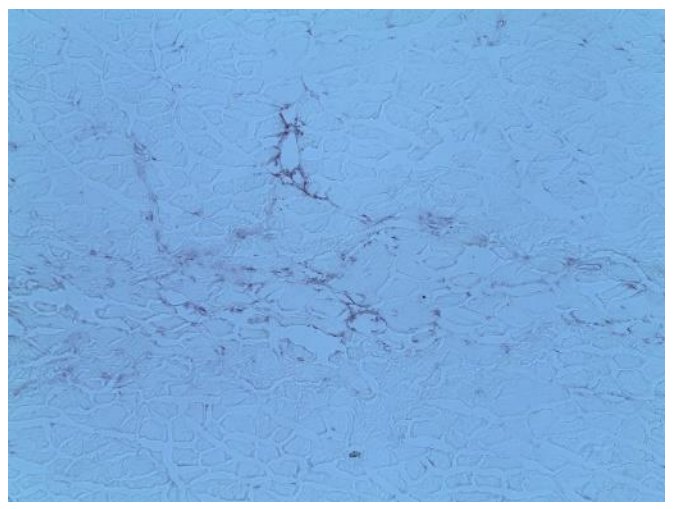

(b)

Figure 13 Selected images

Table 7 Testing detail of special cases for $7 / 4$

\begin{tabular}{|l|l|}
\hline \multicolumn{2}{|c|}{$7 / 4$} \\
\hline Sample selection & From two sample images \\
\hline Class & 2 \\
\hline Positive test sample & 40 \\
\hline Negative sample & 40 \\
\hline
\end{tabular}

From the testing result in Figure 13, it can be shown that the NN classifier has $81.30 \%$ accuracy which is $5 \%$ higher than that of the $\mathrm{CNN}$ classifier. This means that the $\mathrm{NN}$ classifier has higher tolerance of WBC shape inconsistency. 


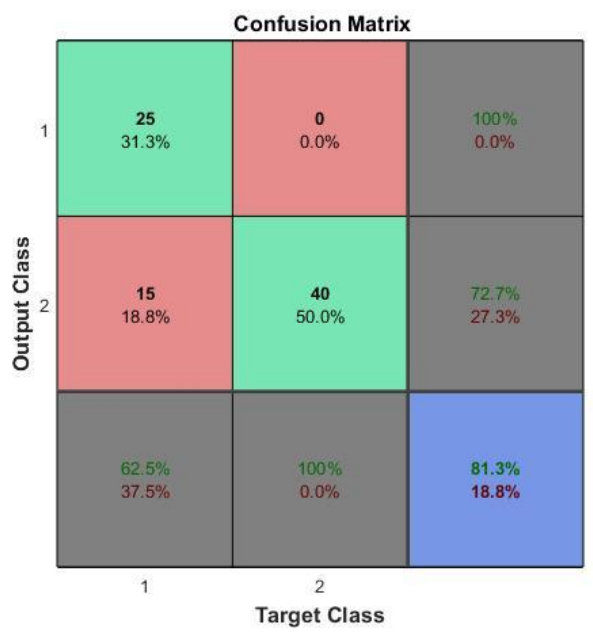

(a)

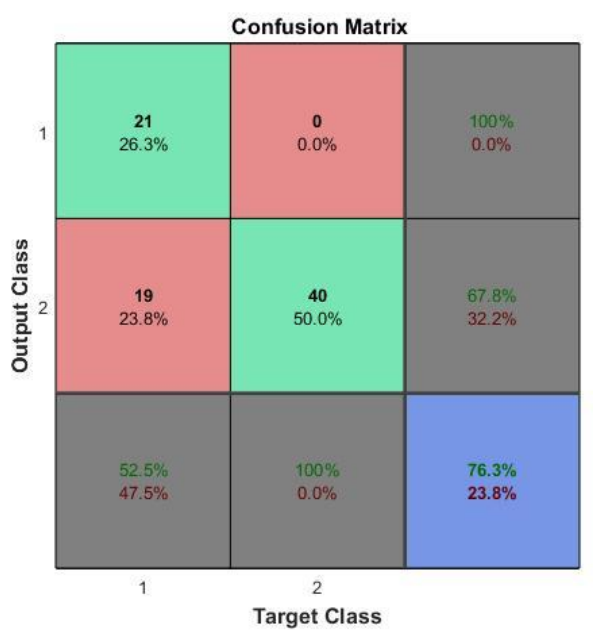

(b)

\section{Figure 14 Testing result of 7/4 special case (a) NN (b) CNN}

\subsubsection{Execution time}

As one important advantage of the CNN classifier, since it extracts features but not simply using fully connected layers, it can shorten the training time extremely especially when the sample amount is huge. In practice, this is validated. However, the NN classifier can achieve a faster detection than the CNN classifier in WBC detection. This test is executed on the computer with i7-6700K (4 cores, 4.00GHz), 8GB RAM and NVIDIA GTX960. As shown in Figure 14, while the NN classifier needs 251.32 s for training, the CNN classifier only consumes 19.08 s, which is 13.17 times faster than NN. This is indeed a significant improvement. However, 251.32s with 3800 training samples is not unacceptable.

The detection applies a sliding window running through the whole image and having a half of the sample size as the step size. In this thesis, the sample size is set as $16^{*} 16$ pixels. Thus the step size is 8 pixels. For detection, the NN classifier needs only 1.16s per image but the CNN classifier takes 
$11.63 \mathrm{~s}$, which means that the CNN classifier is 9.48 times slower than the $\mathrm{NN}$ classifier on detection application. The reason is that convolution and feature extraction consume much more time than multiplication and addition in fully connected layer. As the two-layer NN classifier only contains two fully connected layers, the calculation completes faster. Therefore, for two-class WBC classification, if the detection dataset is huge, the $\mathrm{NN}$ classifier can deliver results faster than the CNN classifier.

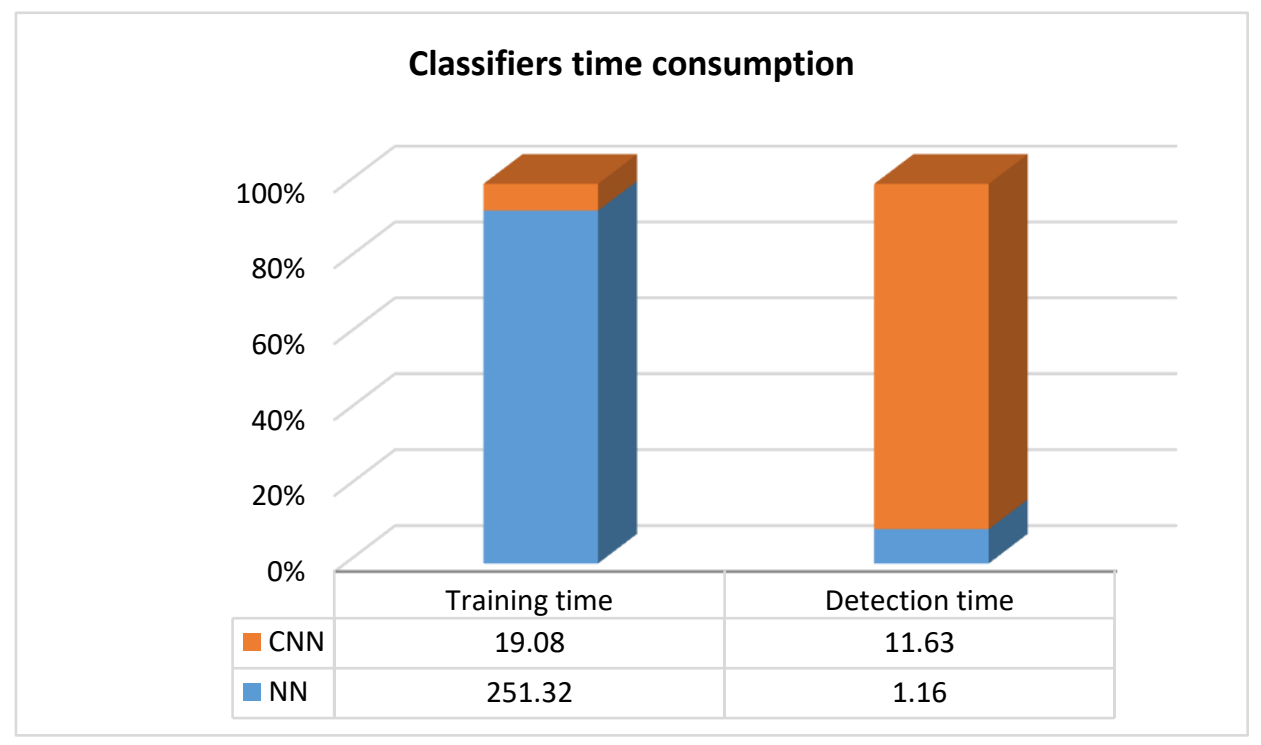

Figure 15 Training and detection time of classifiers

\subsubsection{Effectiveness}

Figure 15 will present the effectiveness of Li Otsu's and LIONN. In Figure 15, (a) shows the original image, (b) shows the LI Otsu's segmentation result, (c) demonstrate LIONN result. We can see that LI Otsu's misses positive WBCs due to the special shape and intensity condition in this image. However, LIONN fixes these false negatives. 


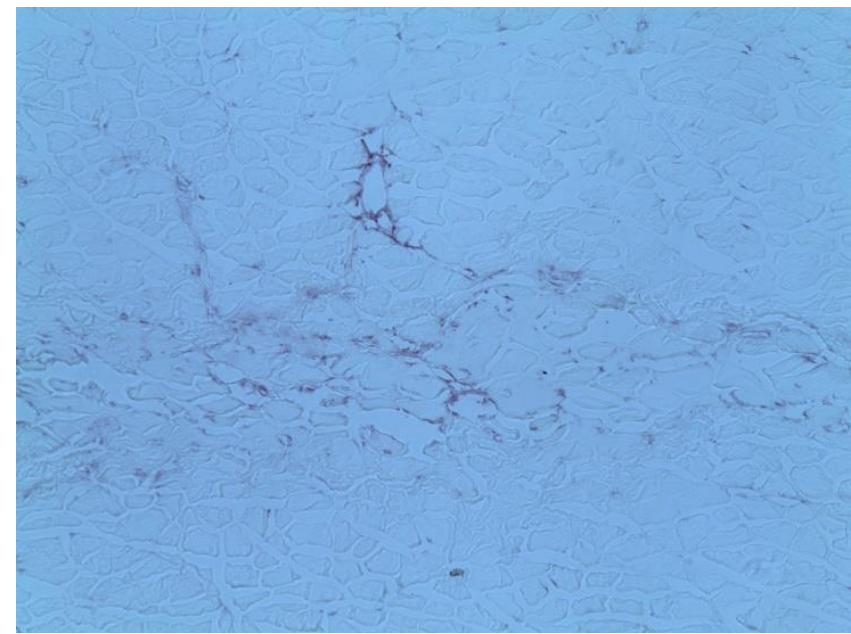

(a)

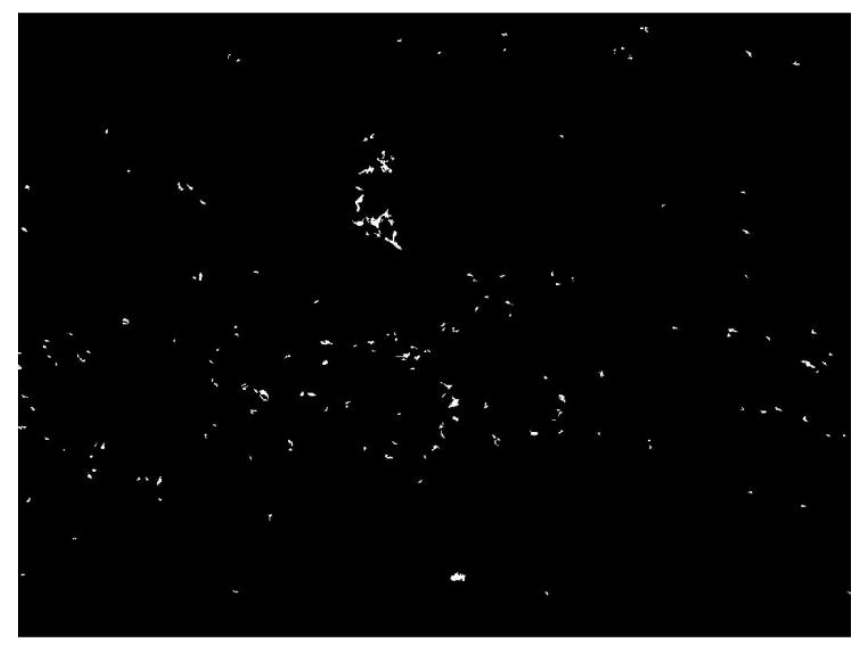

(b)

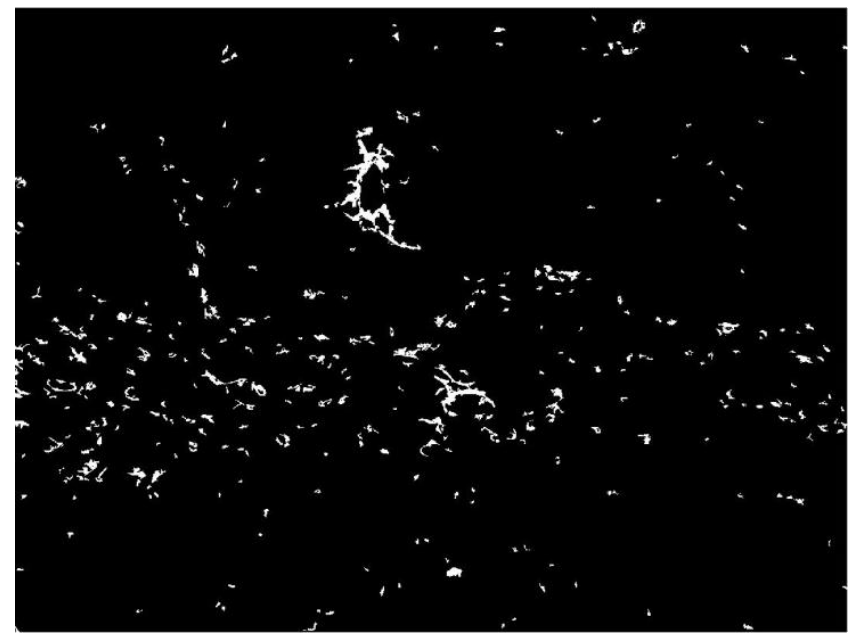

(c)

Figure 16 Effectiveness of LI Otsu's and LIONN (a) original image (b) LI Otsu's segmentation (c) LIONN segmentation 


\section{CHAPTER 6 MUSCLE EDGE DETECTION AND ROI SELECTION}

Muscle edge detection is performed in two steps: muscle texture detection and fuzzy WBC detection. Based on the muscle texture, the muscle edge can be detected. In a situation when WBCs are in high density, the muscle texture could be hardly recognized. Fuzzy classification method allows for refining the result. To accurately detect the muscle edge, the results generated from muscle texture detection and Fuzzy WBC classification are merged. Figure 15 illustrates the steps of the two detectors. The highlighted area shows the necessity of two detections. Due to the densely distributed WBCs in the red rectangle, muscle texture detection returns a result as no texture in this area. By combining fuzzy WBC detection, this error is fixed.

\subsection{Muscle Texture Detection}

Consider a muscle image $f(x, y)$ given in pixel intensity. A step-by-step description of muscle texture detection is given below.

1. Apply $16^{*} 16$ average filter to reduce the appearance of cells and strengthen the muscle fiber texture.

$$
g(x, y)=f(x, y) \times h_{\text {ave }}(x, y)
$$

2. Utilize Gaussian smoothening filter to smooth the image and filter out the noise.

$$
g_{2}(x, y)=g(x, y) \times G_{\sigma}(x, y)
$$




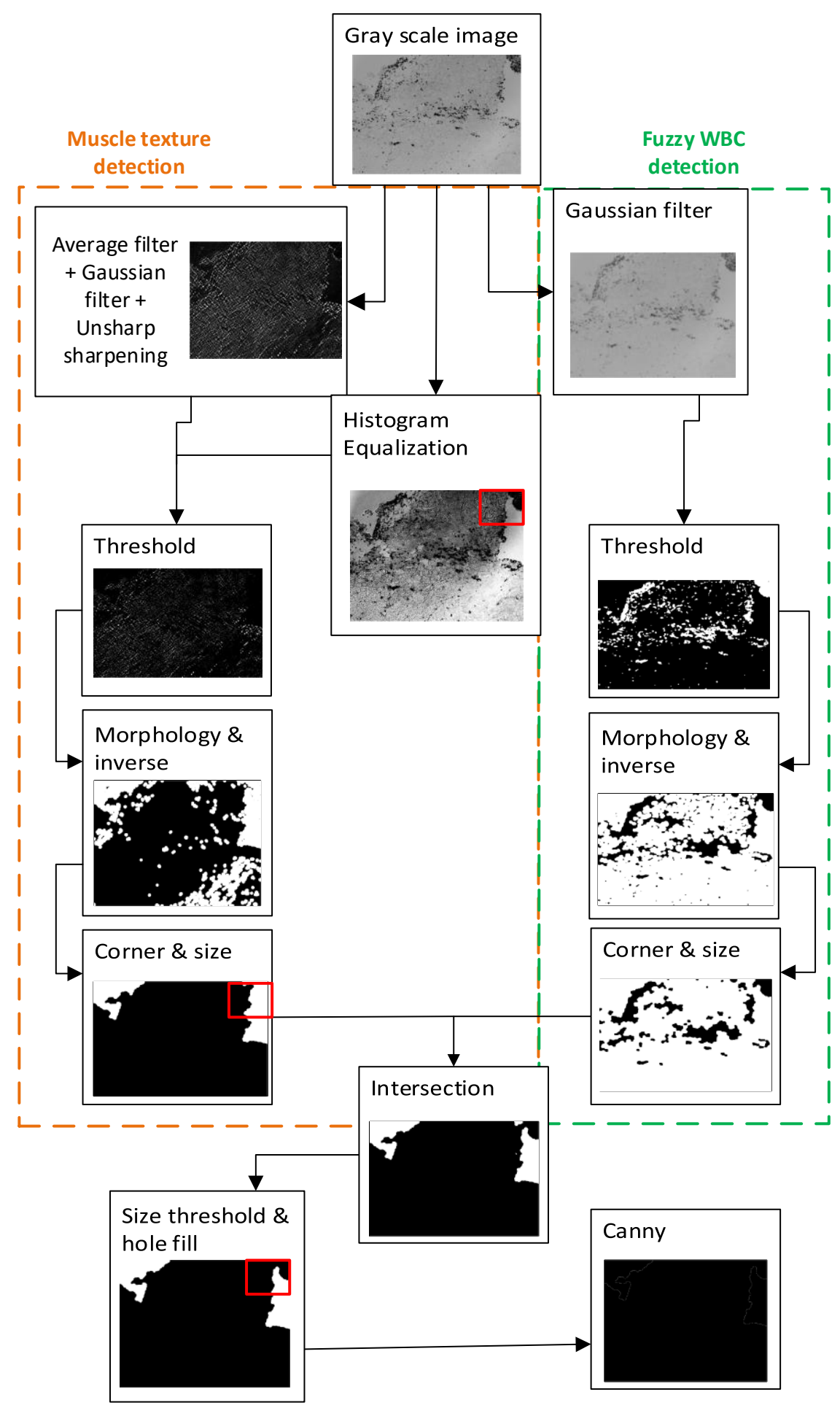

Figure 17 Muscle edge detection scheme 
3. Apply sharpening filter to strengthen texture in muscle area

$$
\begin{gathered}
g_{3}(x, y)=g_{2}(x, y) \times G_{\sigma}(x, y) \\
g_{4}(x, y)=k\left(g_{2}(x, y)-g_{3}(x, y)\right)
\end{gathered}
$$

4. Apply histogram equalization to evenly distribute the intensity of the image.

$$
p_{n}=N_{i} /_{J K}, g_{5}(x, y)=\text { floor }\left((L-1) \sum_{n=0}^{f(x, y)} p_{n}\right)
$$

where $N_{i}$ is the number of pixels with intensity $I, I=0,1, \ldots, L-1 ; J, K$ are the width and length of the input image, floor() rounds down to the nearest integer.

5. Threshold

Standard threshold is applied to $g_{4}$ output image. However, the threshold value $\mathrm{t}$ is obtained using Eq. (4) over $g_{5}$. Let $g_{6}$ be the result of threshold.

$$
g_{6}(x, y)=\left\{\begin{array}{lr}
1 & \text { if } \\
g_{5}(x, y)>t \\
0 & \text { otherwise }
\end{array}\right.
$$

6. Image inverse and morphological closing function.

$$
g_{7}(x, y)=\left[\left(g_{6}{ }^{-1} \ominus b\right) \oplus b\right](x, y)
$$

where $b(x, y)$ is the structuring element.

7. Corner detection to include edges appearing within 20x20 pixel area to any corner of the image. 
Based on image capturing assumption, muscle edge must be laid on the edge or corner of the input image. Therefore, if a candidate does not contain any pixel that is within $20 x 20$ pixel area to any corner of the image, it is excluded. Also, any object that is smaller than 60,000 pixels is excluded.

\subsection{Fuzzy WBC Detection}

The fuzzy WBC detector does not include average filter and sharpening filter as muscle texture detector does. Therefore, this detector is able to capture WBCs and reveal void space (the part of image that does not contain any muscle area). The step-by-step procedure of fuzzy WBC detection is described below.

1. Calculating mean of $f(x, y)$ as:

$$
\text { Mean }=1 /{ }_{N} \sum_{x, y}^{J, K} f(x, y)
$$

where $J, K$ are the width and length of the image, $N$ is the total number of pixels.

2. Apply Gaussian smoothening filter to smooth the image and exclude noise.

$$
g(x, y)=f(x, y) \times G_{\sigma}(x, y)
$$

3. Threshold

Standard Otsu's threshold is applied to $\mathrm{g}(x, y)$ output image. The threshold value is obtained by $k_{1} *$ Mean, where $k_{l}$ is a constant. Let $g_{2}$ be the result of this step.

$$
g_{2}(x, y)=\left\{\begin{array}{c}
1 \text { ifg }(x, y)>k_{1} * \text { Mean } \\
0 \quad \text { otherwise }
\end{array}\right.
$$


4. Perform inverse and morphology transform to expand and connect the effective area

$$
g_{3}(x, y)=\left[\left(g_{2}^{-1} \ominus b\right) \oplus b\right](x, y)
$$

where $b(x, y)$ is the structuring element (disk element is used in this thesis).

5. Corner detection as in Step 7 of the muscle texture detection algorithm.

\subsection{Intersection}

After the results from two detectors are generated, common areas are obtained by intersecting two results. Via excluding small objects and filling holes, the void space is determined. The size threshold is reduced to 50,000 pixels for fine tuning. Large threshold can exclude potential void area. The muscle edge pixel list can be obtained by applying Canny Edge Detector [4], as shown in Figure 15.

\subsection{ROI Selection and Block-Based Analysis}

After muscle edge is detected, any object at distance of 200 pixels to the muscle edge is excluded. The remaining objects are "effective" objects or effective WBCs. The ROI selection is based on the block unit with the block size of 200x200 pixels. The size of 200x200 pixels is chosen based on the size of WBC cluster. Small block size can cut off the clusters, which will cause inaccurate density analysis. For each block, we use scoring to determine if the block is part of the ROI.

$>$ For each block corner, if $d$ (corner $i$, muscle edge) $<200$, score $=$ score +2 .

$>$ For each block, if any effective object exists in this block, score=score +2 . 
$>$ For one block, if the determined void space constitutes more than $50 \%$ of the block area, score $=0$.

By this method, any block that has a score greater than 2 is included in the ROI. From the above, objects obtained by Localized Iterative Otsu's threshold method and located in the ROI are counted. Background (unwanted) objects outside of the ROI will be excluded. We use examples of three images to demonstrate the results of edge detection and ROI selection. In row (6), ROIs are circled by green dash lines. Red spots show the discrete WBCs and blue areas show the clusters of WBCs. Row (7) demonstrates the block histograms. Block histograms depict the amount of blocks that contain certain number of WBCs. The number of WBCs in clusters is estimated via Eq. (3). Therefore, for the uninjured image of mouse number 00311, all 29 effective blocks contains 0-10 WBCs. For 32-hr time point of an injured mouse number 04112, 27 blocks contain 0-10 WBCs, 5 blocks have 11-20 WBCs and 5 blocks have 21-30 WBCs. The image of the injured mouse 15512 at 96-hour point has blocks containing more than 180 WBCs. Thus, block histograms can demonstrate the progress of the cell density distribution in time and space. 
(1)
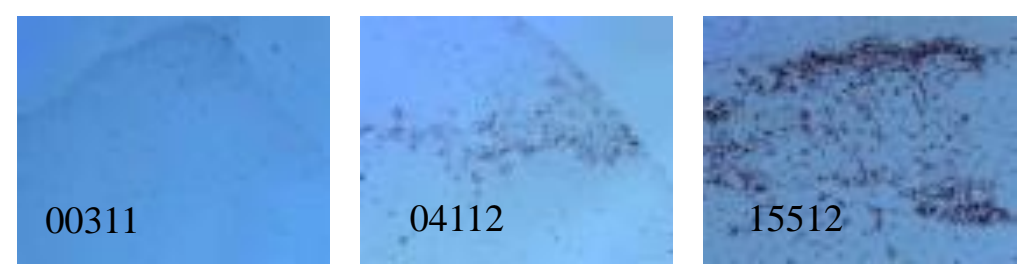

(2)
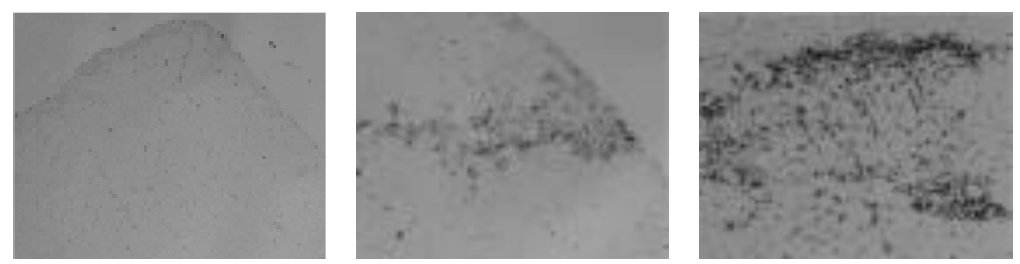

(3)
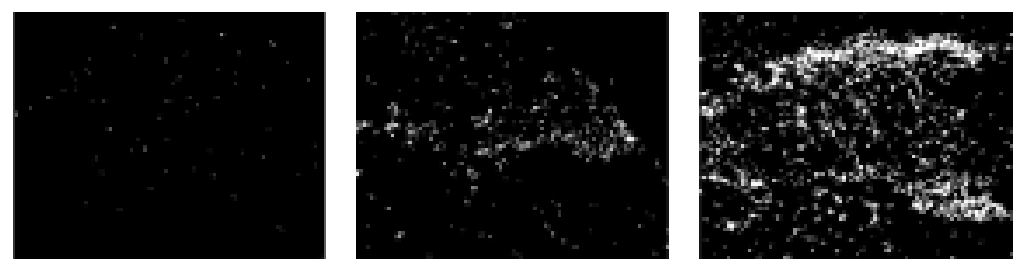

(4)
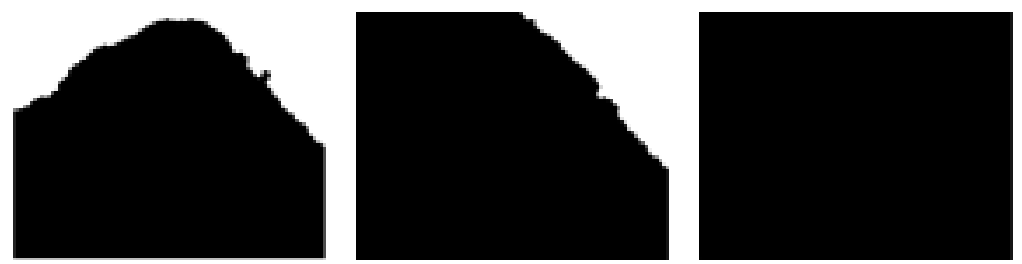

(5)
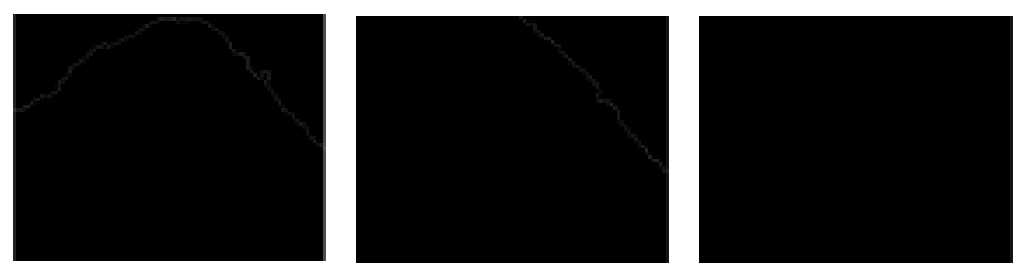

(6)
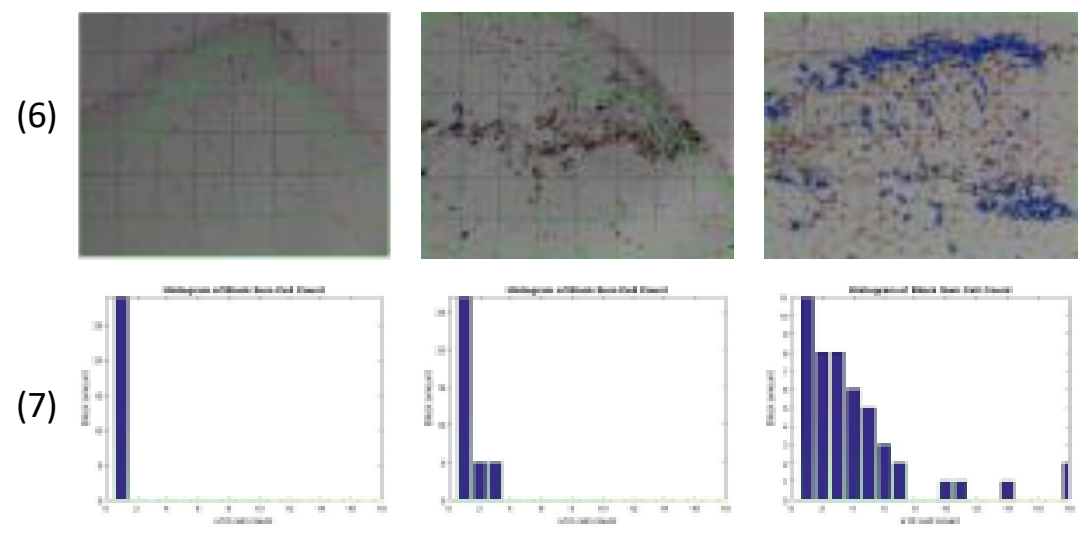

Figure 18 (1) Color image (2) Gray scale image (3) Segmentation result (4) Muscle edge detection (5) Canny muscle edge (6) Composed result \& ROI (7) Block histogram 


\section{CHAPTER 7 EXPERIMENTAL RESULTS}

In this section, we analyze WBCs' expression of the CD68 protein and 7/4 protein. This analysis utilizes images of cross-sections of crush-injured gastrocnemius mouse muscle. Injured and contralateral uninjured muscles were collected at 4, 8, 24, 32, 48, 96 and 192 hours after the injury and then frozen. Each frozen muscle was sliced into 10-micron thick sections. Serial sections including both injured and uninjured muscles were stained with an antibody that recognizes CD68-positive WBCs or 7/4-positive WBCs. Images were captured under 100X magnification using Image-Pro software. Also, a group of images were obtained from muscles of mice that did not undergo crush injury.

The quantification results obtained by the LI Otsu's threshold, LI Otsu's method assisted with the NN classifier (LIONN), and LI Otsu's method assisted with the CNN classifier (LIOCNN) for CD68 and 7/4 images will be discussed. The overall WBC amount, discrete cell amount, cluster amount and density distribution of CD68-positive and 7/4-positive WBCs will be illustrated.

\subsection{Comparison of Three Methods}

\subsubsection{Results of CD68}

Due to different area of ROI across images, data are normalized by unit area (10,000 sq.pixels).

From Figure 17 we can see that, for three methods, both overall amount of WBC and normalized WBC amount are close. Because of the well dyeing of CD68 images, all three methods have results with a slight difference. From Figure 10, we can see that the CNN classifier has $1.2 \%$ error rate. 
Therefore, with the correction of the CNN classifier, this result can be considered as reliable for CD68 images.

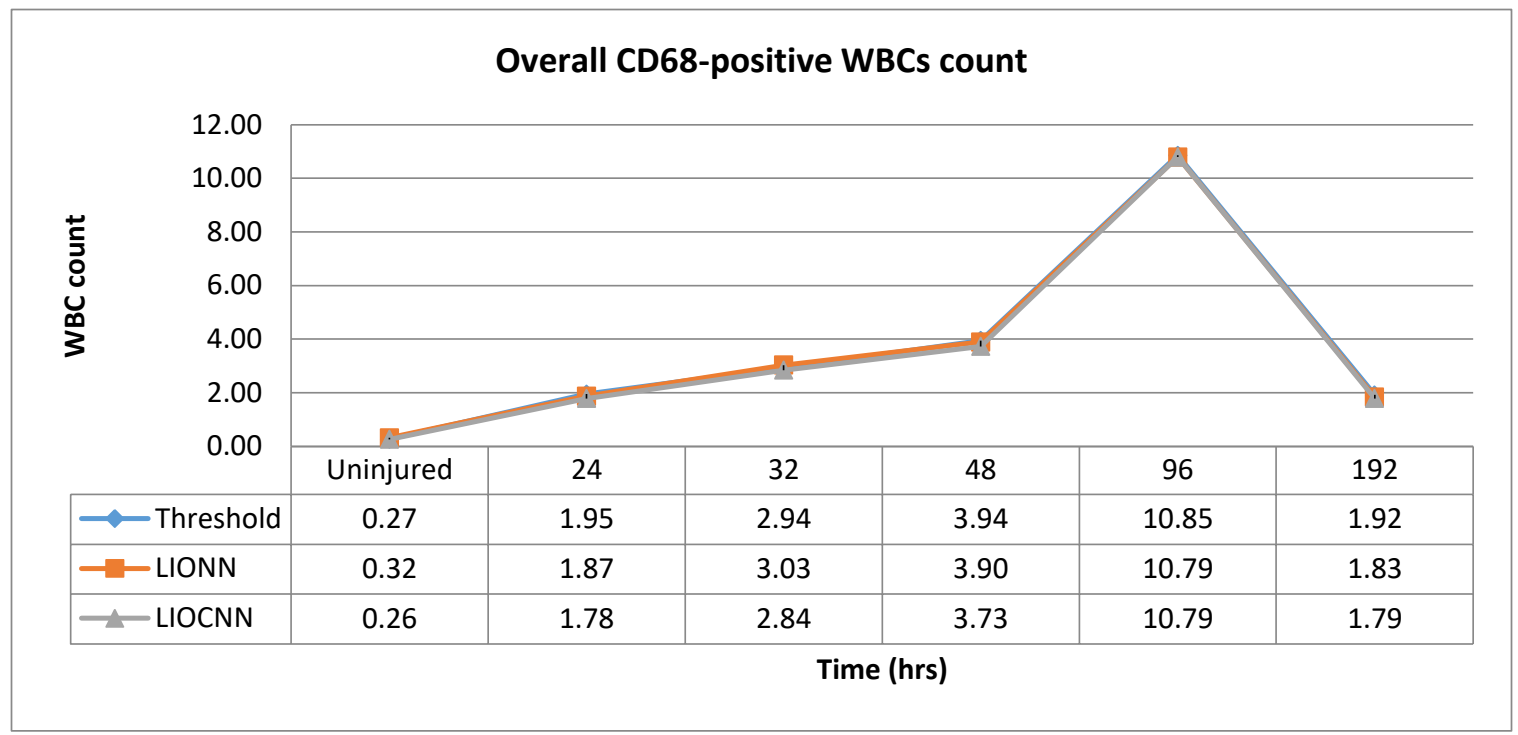

Figure 19 Overall amount of CD68-positive WBCs

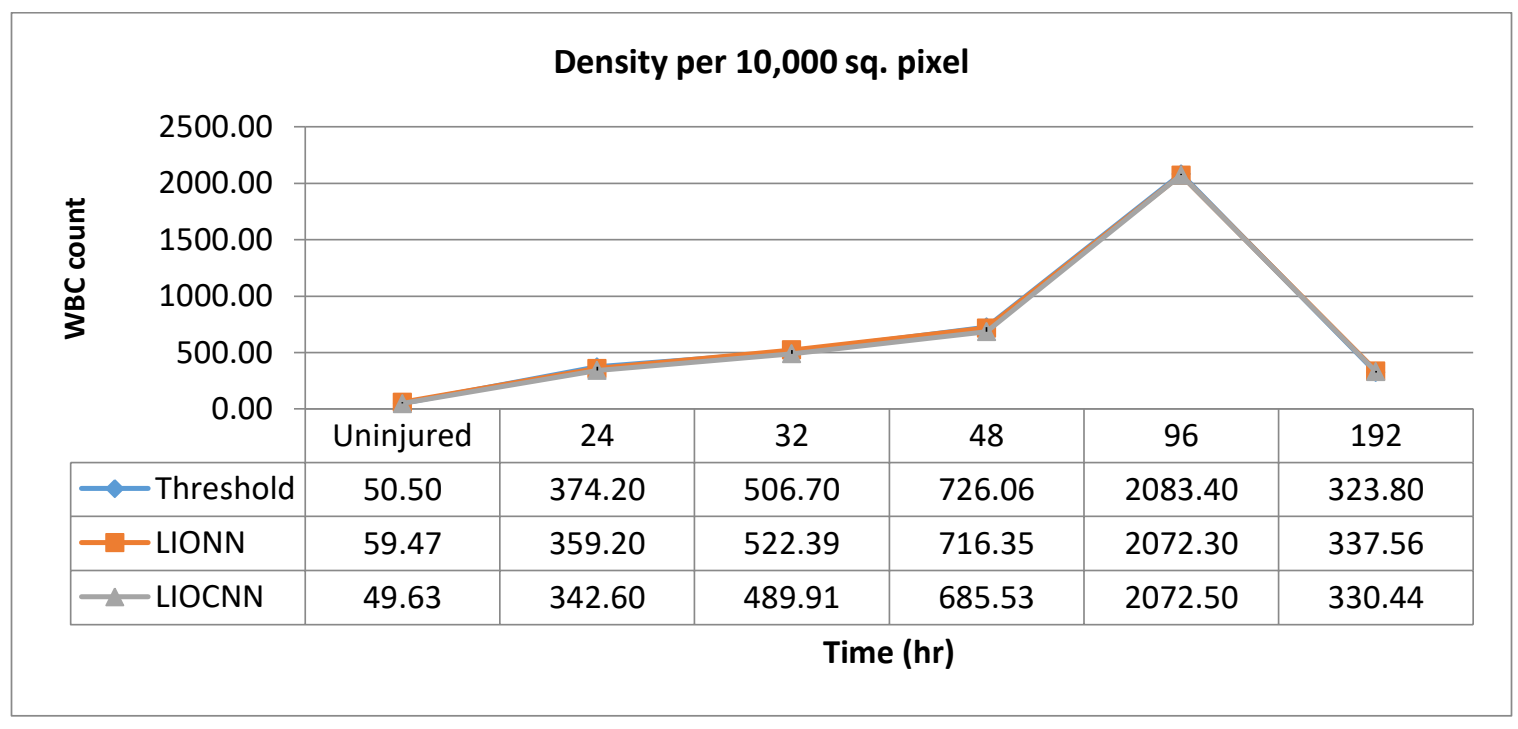

Figure 20 Density results of CD68-positive WBCs

\subsubsection{Results of $7 / 4$}

From Figure 19 we can see that LI Otsu's threshold detects a larger amount of WBCs than LIONN 
and CLIONN, which is due to false positives. As Figures 10 and 13 show, although CNN has a $0.5 \%$ higher overall accuracy, the NN classifier has 5\% higher accuracy for complicated cases. And these cases occur frequently at early time points. Therefore, LIONN can be considered to have the best result for $7 / 4$ images.

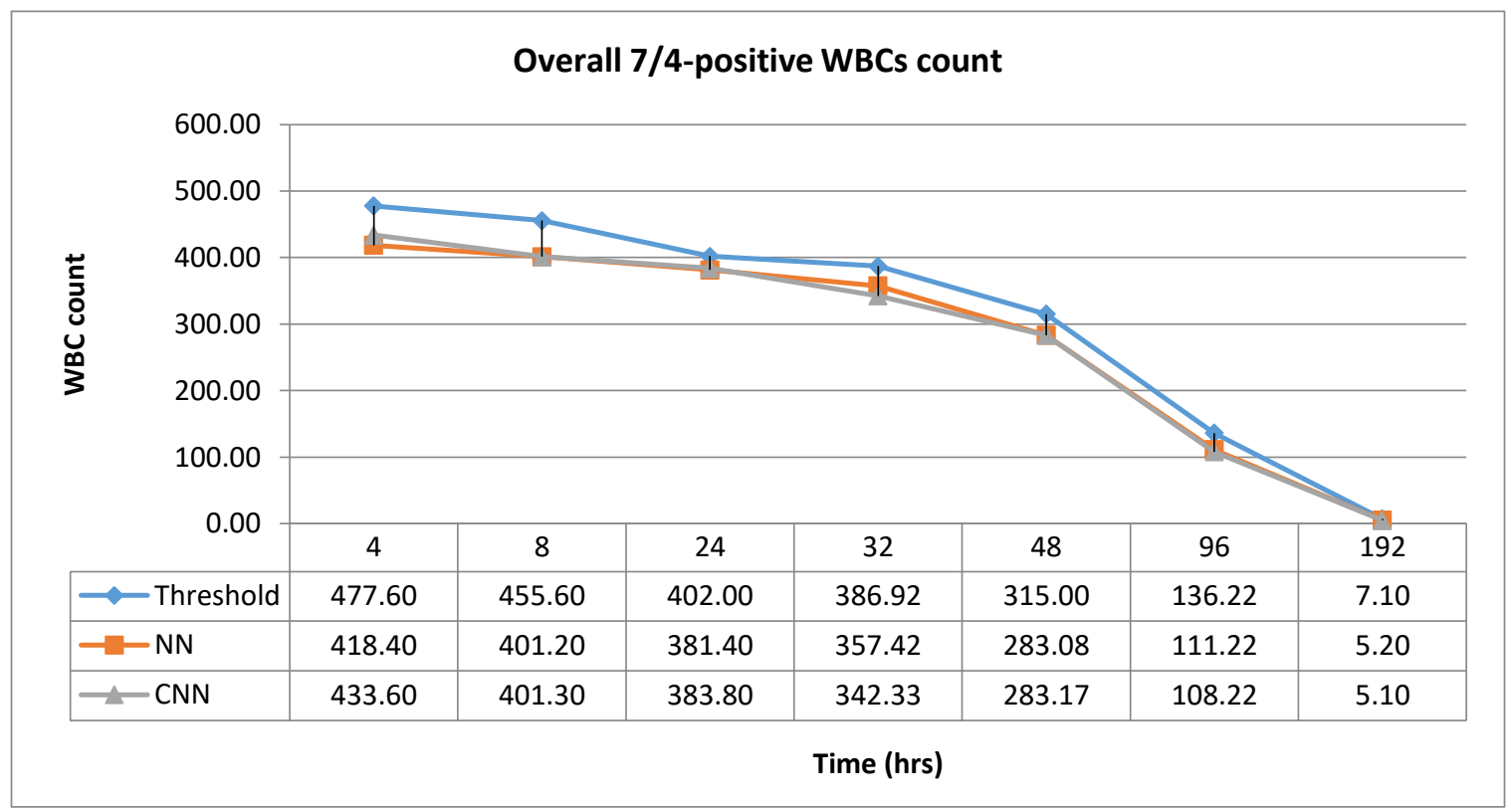

Figure 21 Overall amount of 7/4-positive WBCs

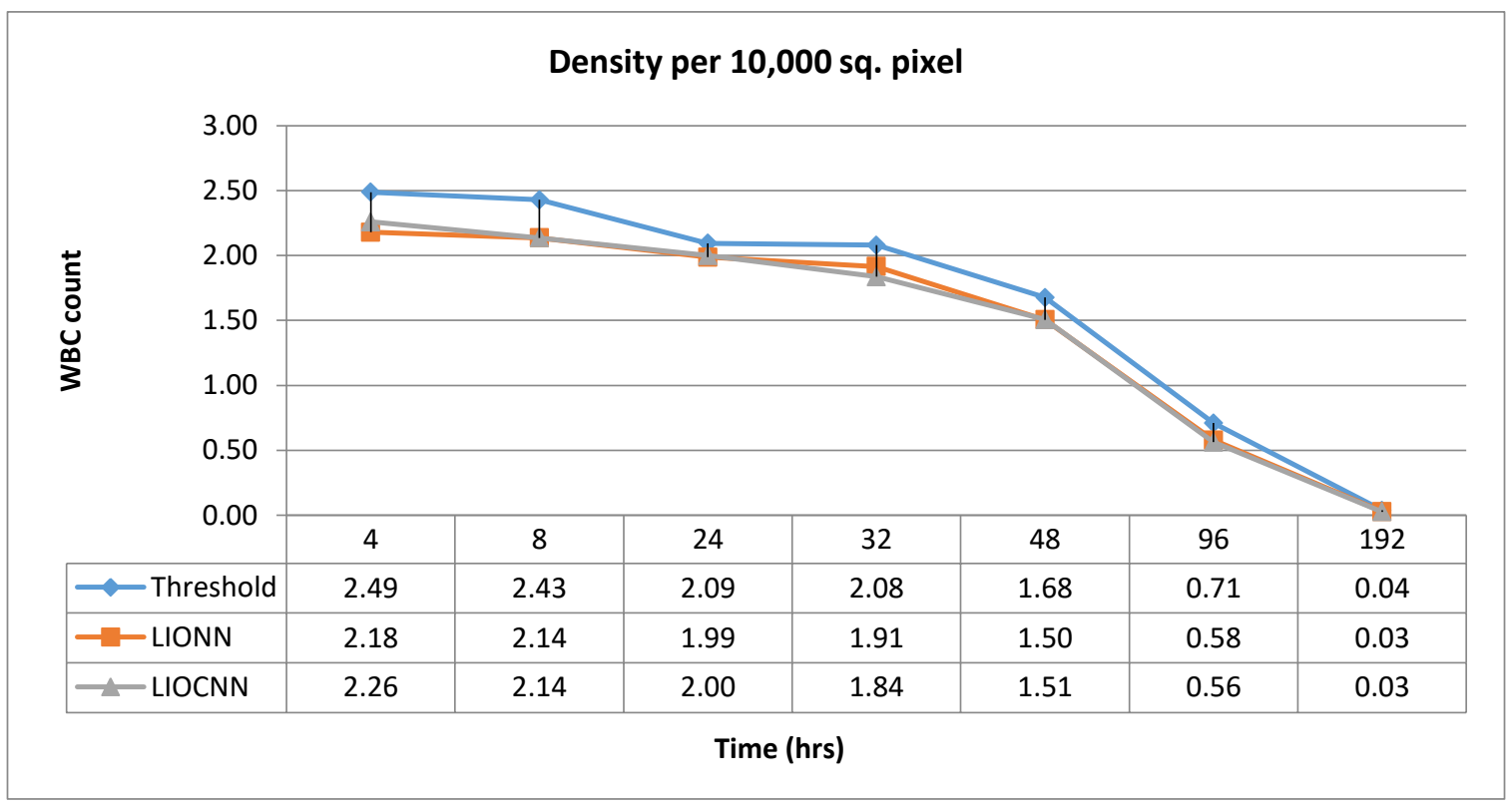

Figure 22 Density results of 7/4-positive WBCs 


\subsection{Amount of WBCs and Clusters}

\subsubsection{Results of CD68 images}

The whole proposed framework is implemented in MATLAB. Totally 95 images including 65 injured muscle images and 30 uninjured muscle images of CD68 are processed automatically. Features presented in Section 3.5 are extracted and analyzed using LIOCNN, with the averaged results shown in Table 8 .

Table 8 Analysis result of CD68-positive WBCs using LIOCNN

\begin{tabular}{|l|l|l|l|l|l|l|}
\hline per image & $\begin{array}{l}\text { \# of } \\
\text { Discrete } \\
\text { WBCs }\end{array}$ & $\begin{array}{l}\text { \# of } \\
\text { Clustered } \\
\text { WBCs }\end{array}$ & $\begin{array}{l}\text { \# of } \\
\text { WBCs in } \\
\text { clusters }\end{array}$ & $\begin{array}{l}\text { Size of } \\
\text { Discrete } \\
\text { WBCs }\end{array}$ & $\begin{array}{l}\text { Overall } \\
\text { \# of } \\
\text { WBCs }\end{array}$ & \#/10,000p \\
\hline Uninjured & 47.43 & 0.10 & 2.20 & 64.43 & 49.63 & 0.26 \\
\hline $24-\mathrm{hr}$ & 278.60 & 5.80 & 64.00 & 89.31 & 342.60 & 1.78 \\
\hline $32-\mathrm{hr}$ & 306.65 & 15.22 & 183.26 & 103.08 & 489.91 & 2.84 \\
\hline $48-\mathrm{hr}$ & 364.18 & 28.53 & 321.35 & 113.87 & 685.53 & 3.73 \\
\hline 96-hr & 561.10 & 44.30 & 1511.40 & 108.02 & 2072.50 & 10.79 \\
\hline 192-hr & 234.89 & 4.44 & 95.56 & 80.62 & 330.44 & 1.79 \\
\hline
\end{tabular}

From Table 8, we can see that 48.13 discrete CD68-positive WBCs and only 0.1 clusters are contained in uninjured muscles. After the injury, CD68-positive WBCs and their clusters are reaching a peak at 96-hr. After 96-hr, both discrete and clustered CD68-positive WBC counts decrease. Figures 21 and 22 show the trend. 


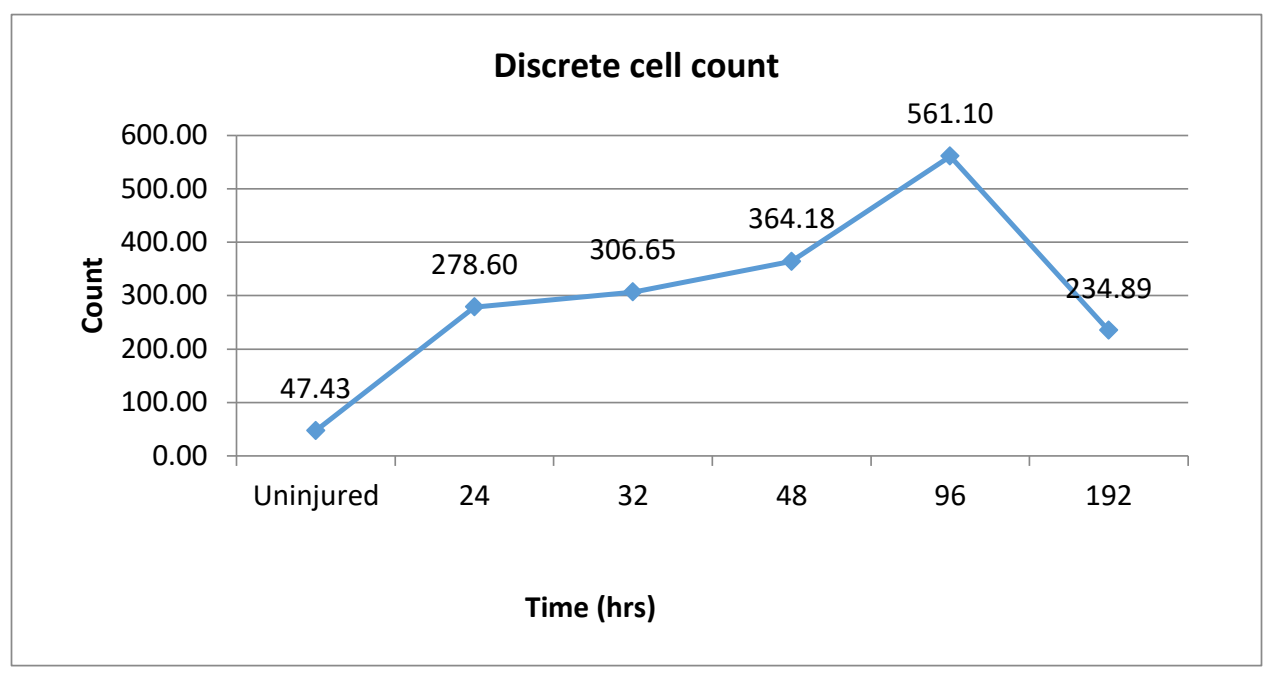

Figure 23 Amount of discrete CD68-positive WBCs

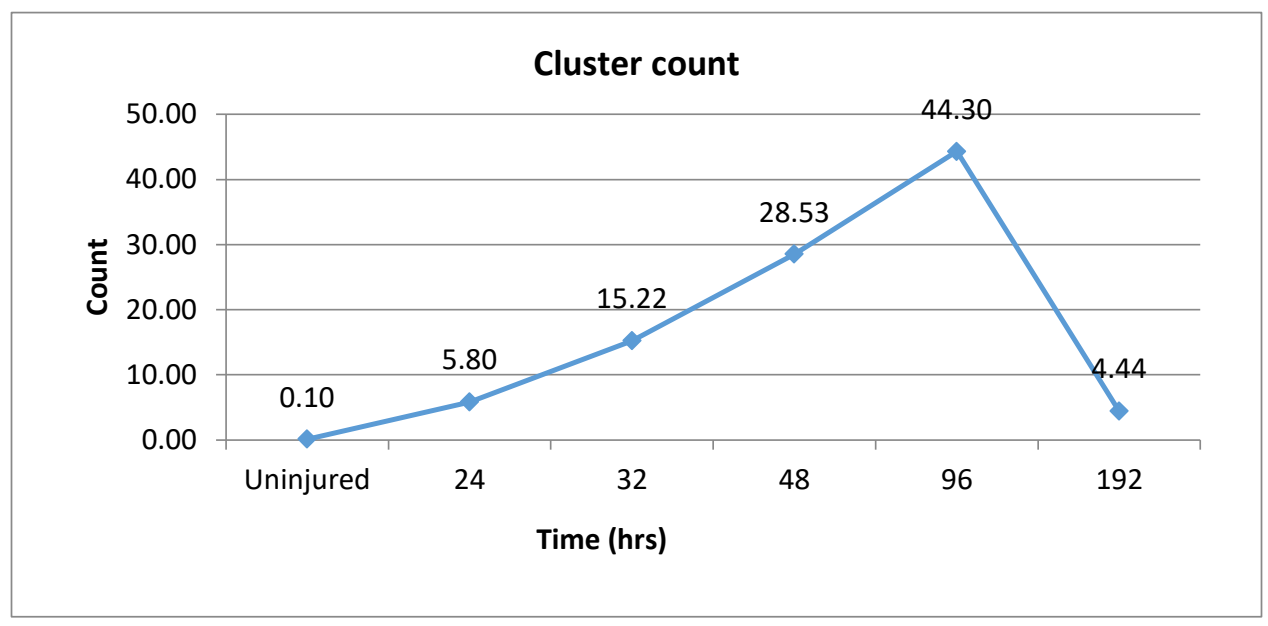

Figure 24 Amount of clusters of CD68-positive WBCs

\subsubsection{Results of $7 / 4$ images}

Totally 63 injured 7/4 images are studied and processed by the proposed framework. These images are from 4-hr to 192-hr after injury. Their features are extracted and analyzed using LIONN. As shown in Table 9, the amount of discrete WBCs fluctuates within 40 at time points 4-hr, 8-hr, 24-hr and 32-hr, and keeps decreasing after 32-hr. 
Table 9 Analysis result of 7/4-positive WBCs using LIONN

\begin{tabular}{|l|l|l|l|l|l|l|}
\hline $\begin{array}{l}\text { per } \\
\text { image }\end{array}$ & $\begin{array}{l}\text { \# of } \\
\text { Discrete } \\
\text { WBCs }\end{array}$ & $\begin{array}{l}\text { \# of } \\
\text { Clustered } \\
\text { WBCs }\end{array}$ & $\begin{array}{l}\text { \# of WBCs } \\
\text { in clusters }\end{array}$ & $\begin{array}{l}\text { Size of } \\
\text { Discrete } \\
\text { WBCs }\end{array}$ & $\begin{array}{l}\text { Overall } \\
\text { \# of } \\
\text { WBCs }\end{array}$ & \#/10,000p ${ }^{2}$ \\
\hline 4-hr & 322.60 & 9.60 & 95.80 & 90.76 & 418.40 & 2.18 \\
\hline $8-\mathrm{hr}$ & 300.40 & 9.40 & 100.80 & 100.16 & 401.20 & 2.14 \\
\hline $24-\mathrm{hr}$ & 335.40 & 5.00 & 46.00 & 90.63 & 381.40 & 1.99 \\
\hline $32-\mathrm{hr}$ & 296.50 & 5.08 & 60.92 & 88.61 & 357.42 & 1.91 \\
\hline $48-\mathrm{hr}$ & 246.67 & 4.50 & 36.42 & 82.63 & 283.08 & 1.50 \\
\hline $96-\mathrm{hr}$ & 103.78 & 0.89 & 7.44 & 73.72 & 111.22 & 0.58 \\
\hline 192-hr & 5.20 & 0.00 & 0.00 & 86.49 & 5.20 & 0.03 \\
\hline
\end{tabular}

However, the amount of clusters remains the same value at 4-hr and 8-hr and then decreases. Then it remains at a value again at 24-hr, 32-hr and 48-hr. After 48-hr, the amount of clusters decreases.

Figures 23 and 24 show this trend.

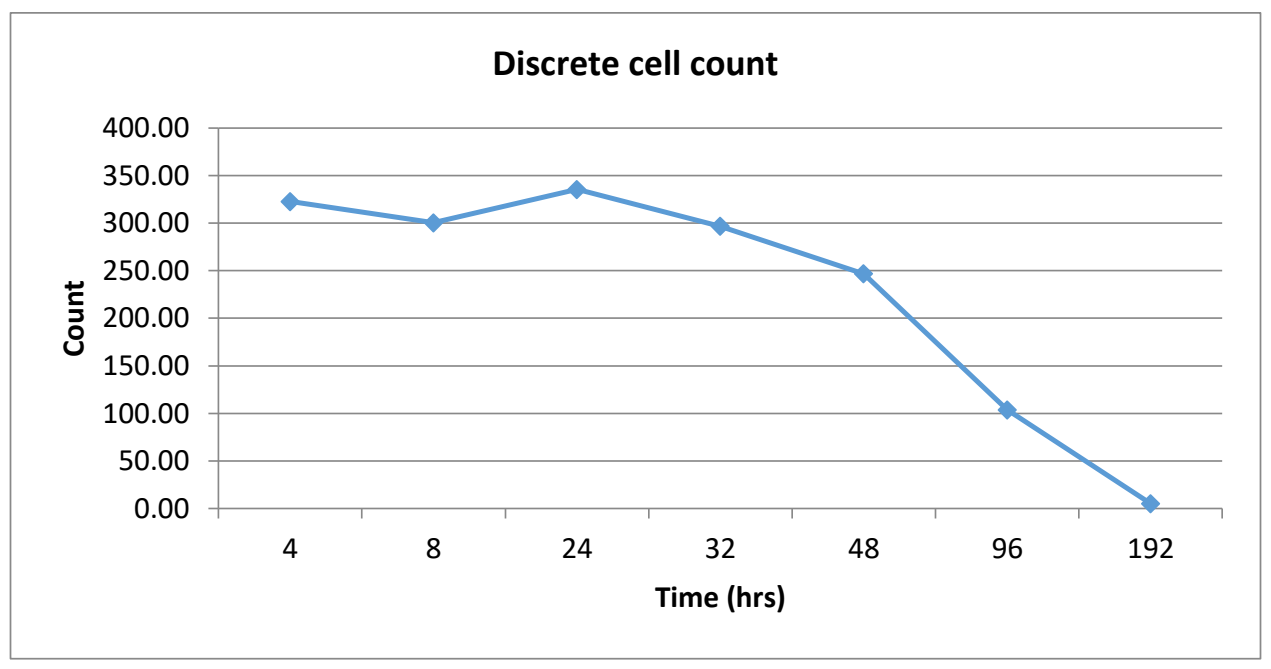

Figure 25 Amount of discrete 7/4-positive WBCs 


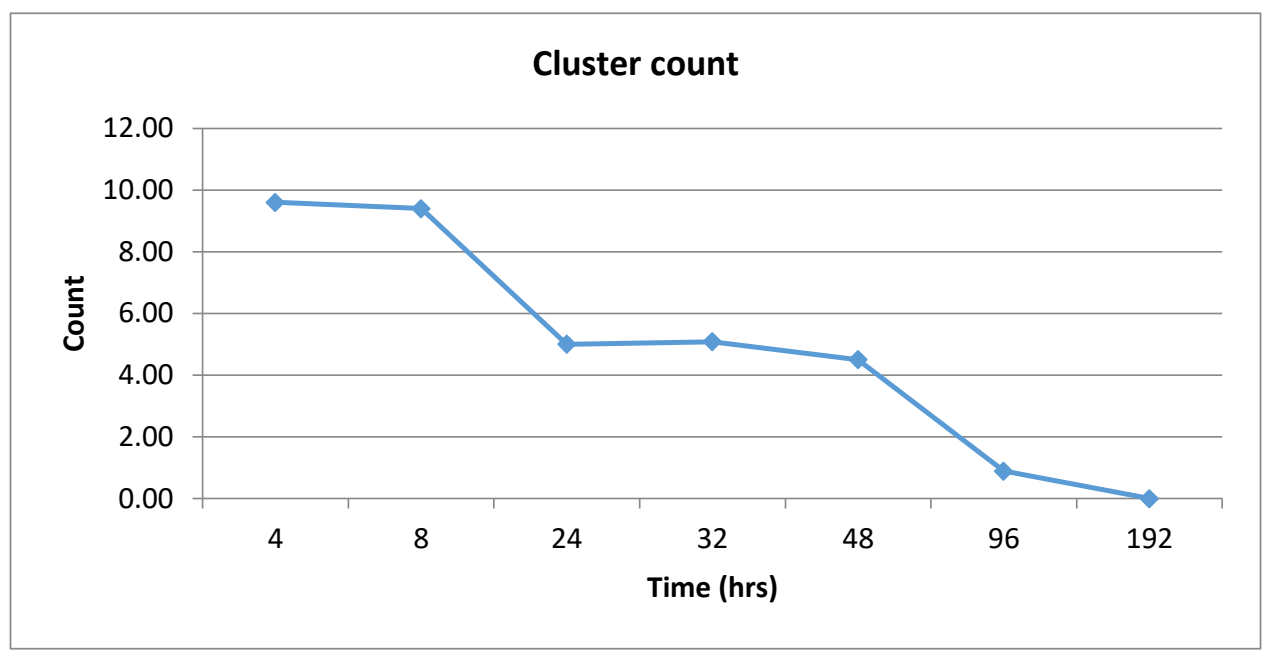

Figure 26 Amount of clusters of 7/4-positive WBCs

\subsection{Density Distribution}

\subsubsection{Results of CD68 images}

After applying Eq. (3), an estimate of CD68-positive WBC count in clusters and the overall CD68-positive WBC count are obtained (as shown in Table 8). The density distribution of CD68-positive WBCs is represented by the binned histogram: the number of blocks containing $0 \sim 20,21 \sim 40, \ldots,>160$ cells are selected as the representative bin values. The histogram of CD68-positive WBC counts per block is shown in Figure 9. Figure 10 shows the log scale histogram for the visualization purpose. The tabulated values using Eq. (8) are presented in Fig.11.

$$
N_{\text {block }}{ }^{\prime}=\log \left(10 N_{\text {block }}+1\right)
$$

As shown in Figure 7 (c), in uninjured muscles, over 99\% blocks contain only from 0 20 CD68-positive WBCs. After the injury, the number of blocks that contain more than 20 cells increases. At 96-hr after the injury, the block histogram demonstrates its variety from 0-20 to >160, 
which represents the increasing density of CD68-positive WBCs from uninjured case to 96-hr after injury. Consistent with the trend in Figure 7 (c) and (d), the peak density occurs at 96-hr. The density of WBCs decreases between 96-hr and 192-hr, that is reflected by the decrease of the number of blocks containing more than 20 cells. At 192-hr after injury, the WBC count is still higher than the uninjured condition. Figure 25 demonstrates the average WBC amount per 10,000 sq. pixel. It shows the trend of density change which can be cross-proved by Figures 26 and 27 .

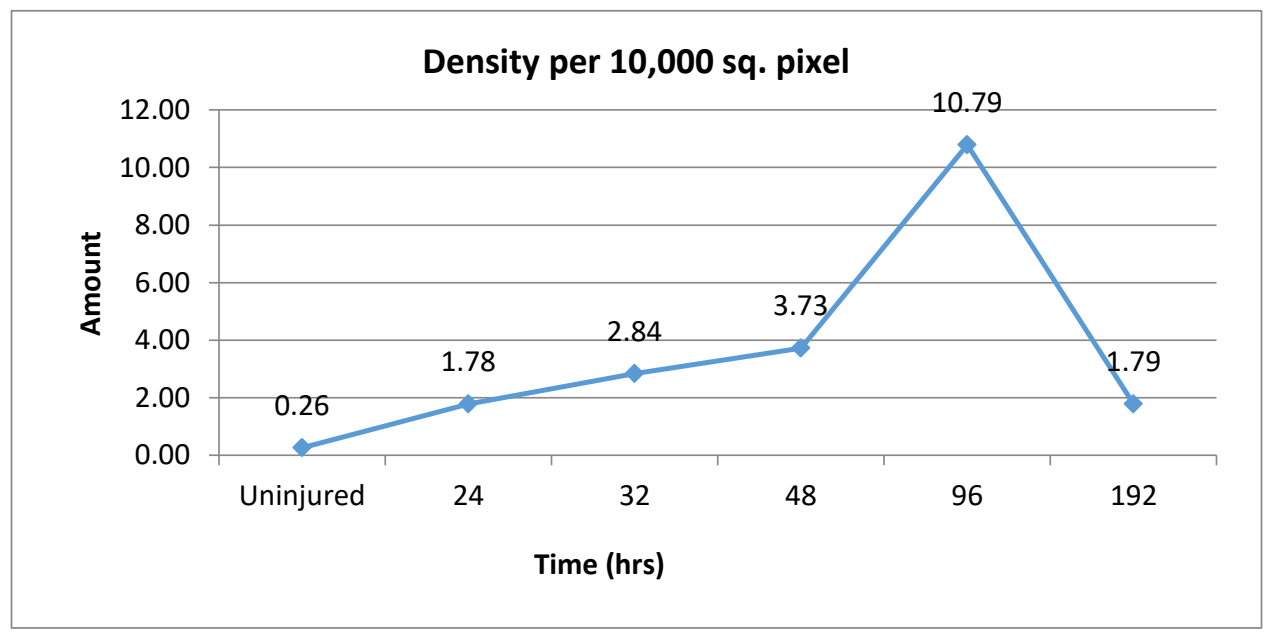

Figure 27 Density result of CD68-positive WBCs 


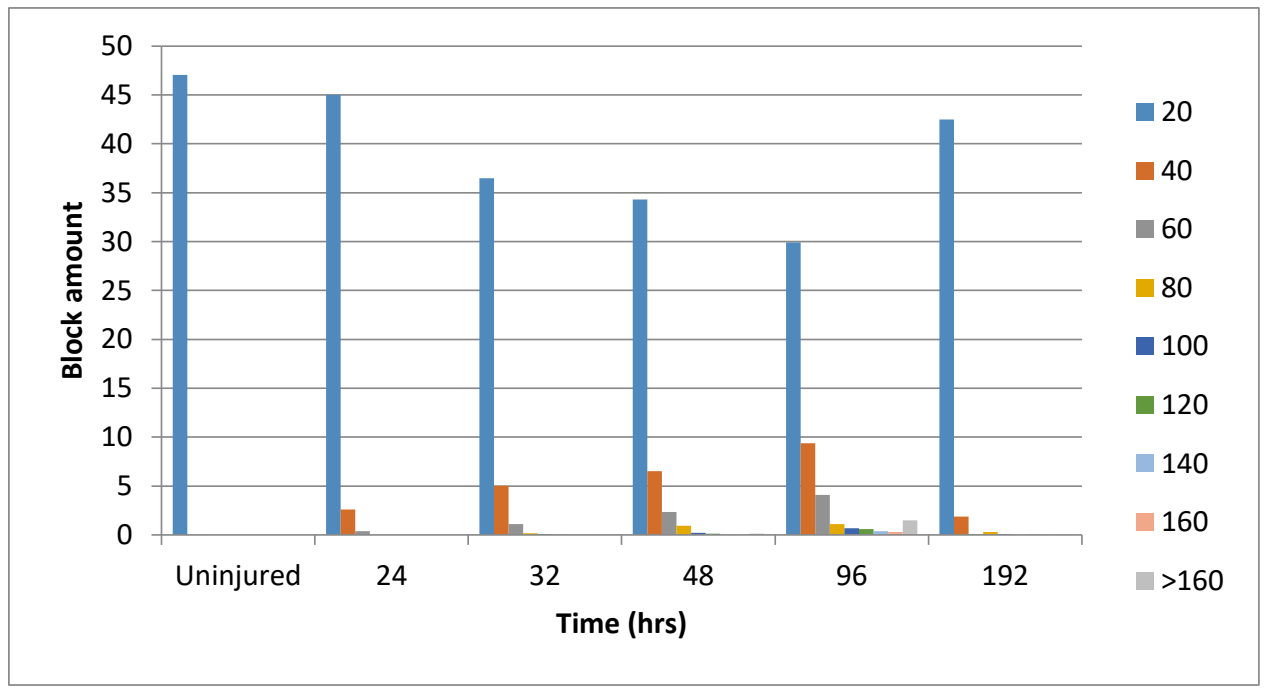

Figure 28 Histogram of CD68-positive WBCs per block

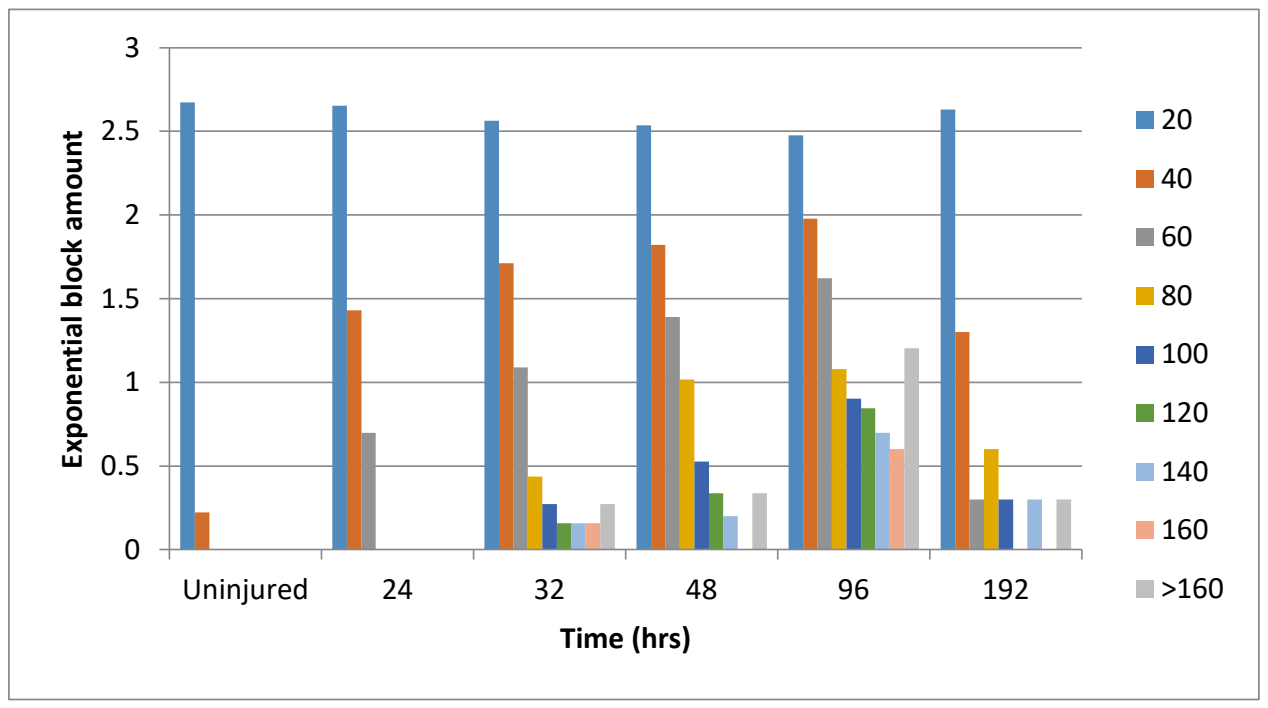

Figure 29 Exponential histogram of CD68-positive WBCs per block

\subsubsection{Results of $7 / 4$ images}

As the same method applied to CD68 images, block histogram and average density are analyzed.

Different with CD68, 7/4 demonstrates a basically consistent density at 4-hr, 8-hr, 24-hr and 32-hr.

From Figure 28, one can see a $27 \%$ decrease at 48 -hr after injury. After 48 -hr, the density of $7 / 4$ decreases till 0.03 per 10,000 sq. pixel at 192-hr after injury, which can be seen as no cell. Figure 28 
demonstrates a numeric result. However, due to the limitation of sample size (4-hr and 24-hr both include 5 samples), this result can fluctuate with sample size increasing. Since the histogram of block can be used to analyze the distribution of density on a range, it can be believed as a more appropriate method to present the result. Figure 30 is generated by applying Eq. (8) to the data of Figure 29. We can see that from 4-hr to 48-hr, the density of WBC has a slight decrease, but basically remains at the same level. And the density drops fast after 48-hr.

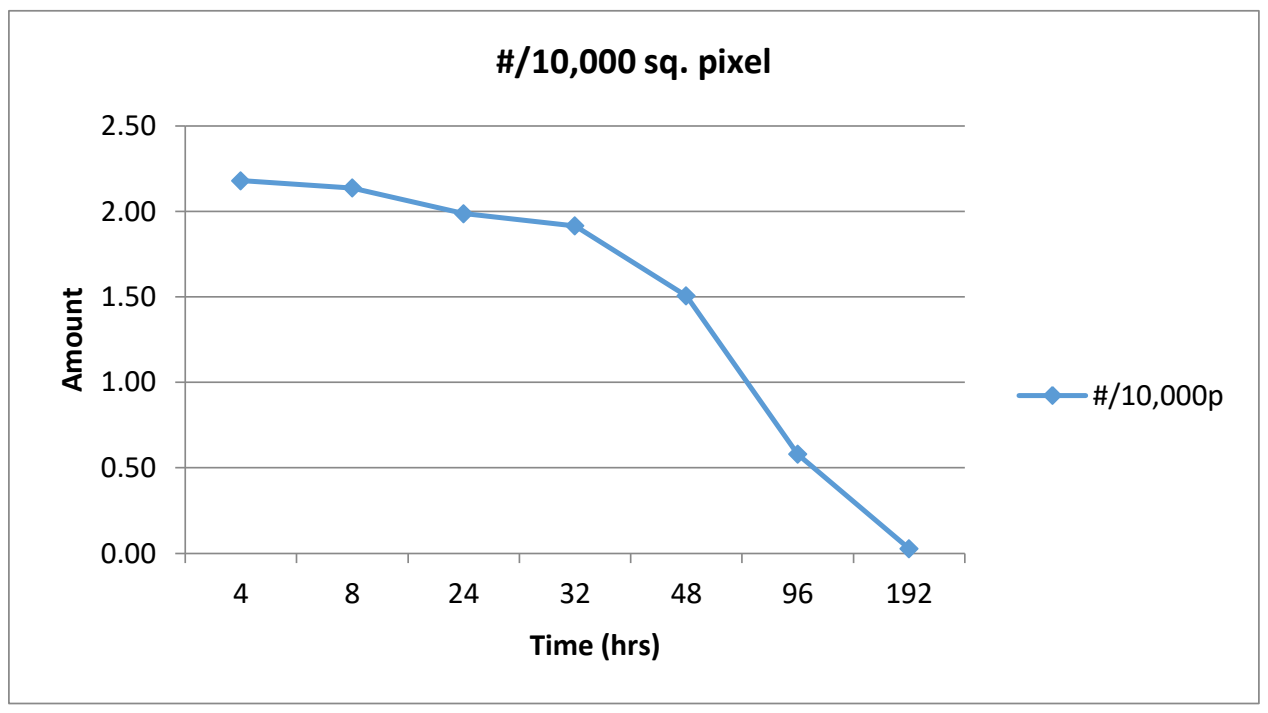

Figure 30 Density result of 7/4-positive WBCs 


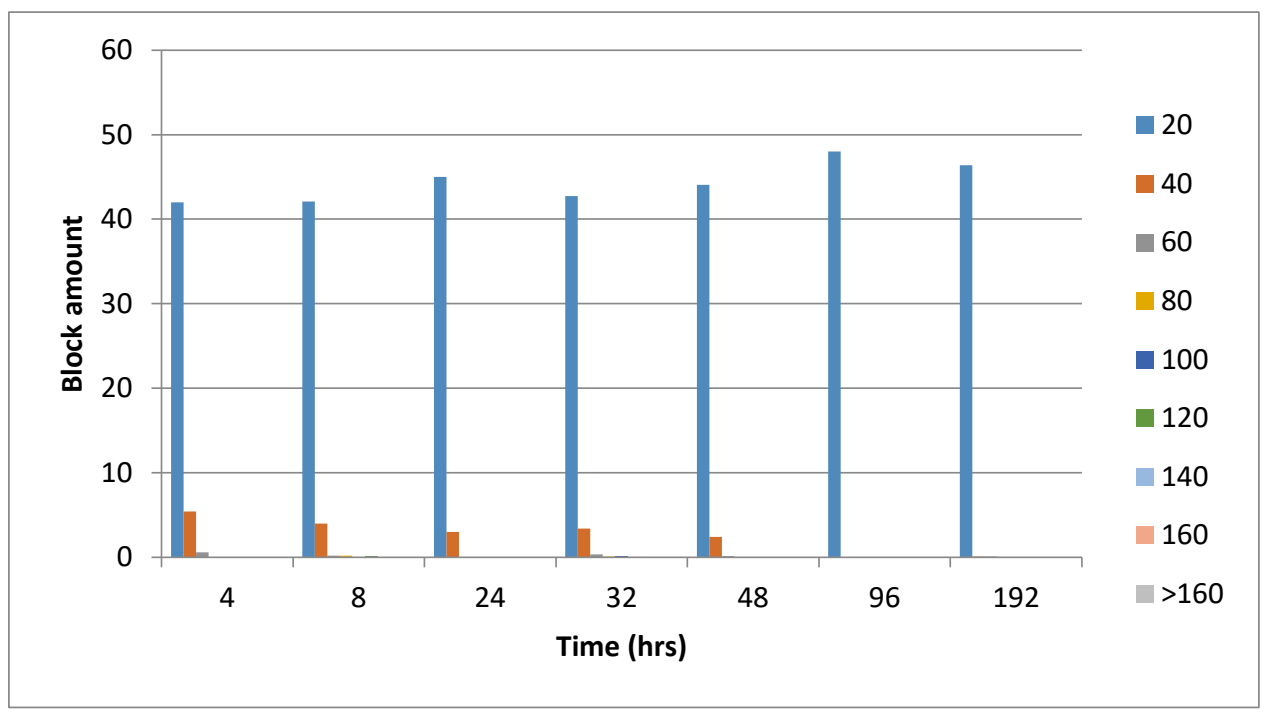

Figure 31 Histogram of 7/4-positive WBCs per block

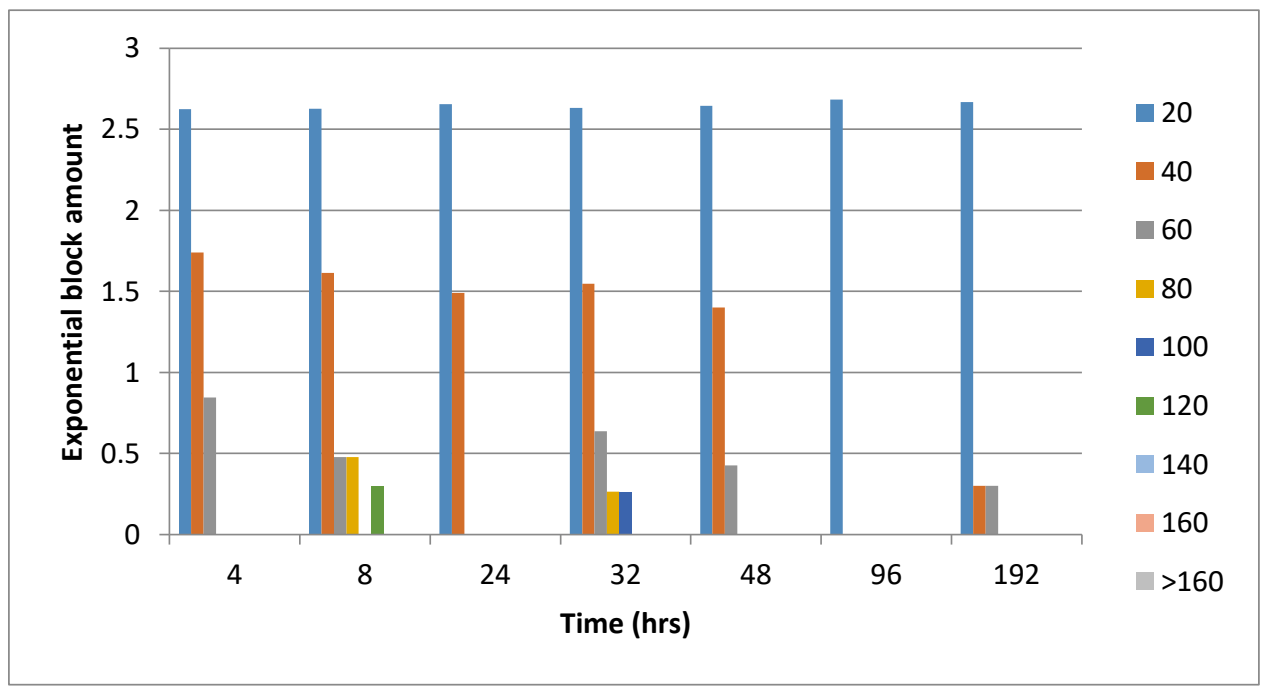

Figure 32 Exponential histogram of 7/4-positive WBCs per block

\subsection{WBC Model in Muscle Healing Process}

As discussed above, the count and density of CD68 and 7/4 have been presented. If expand the axis to unit length, we can see the trend of change. As shown in Figure 33, CD68 has basically linear increasing from uninjured condition to $96-\mathrm{hr}$ after injury. For $7 / 4$, its decrease is linear as well from 4-hr after injury to 192-hr. 


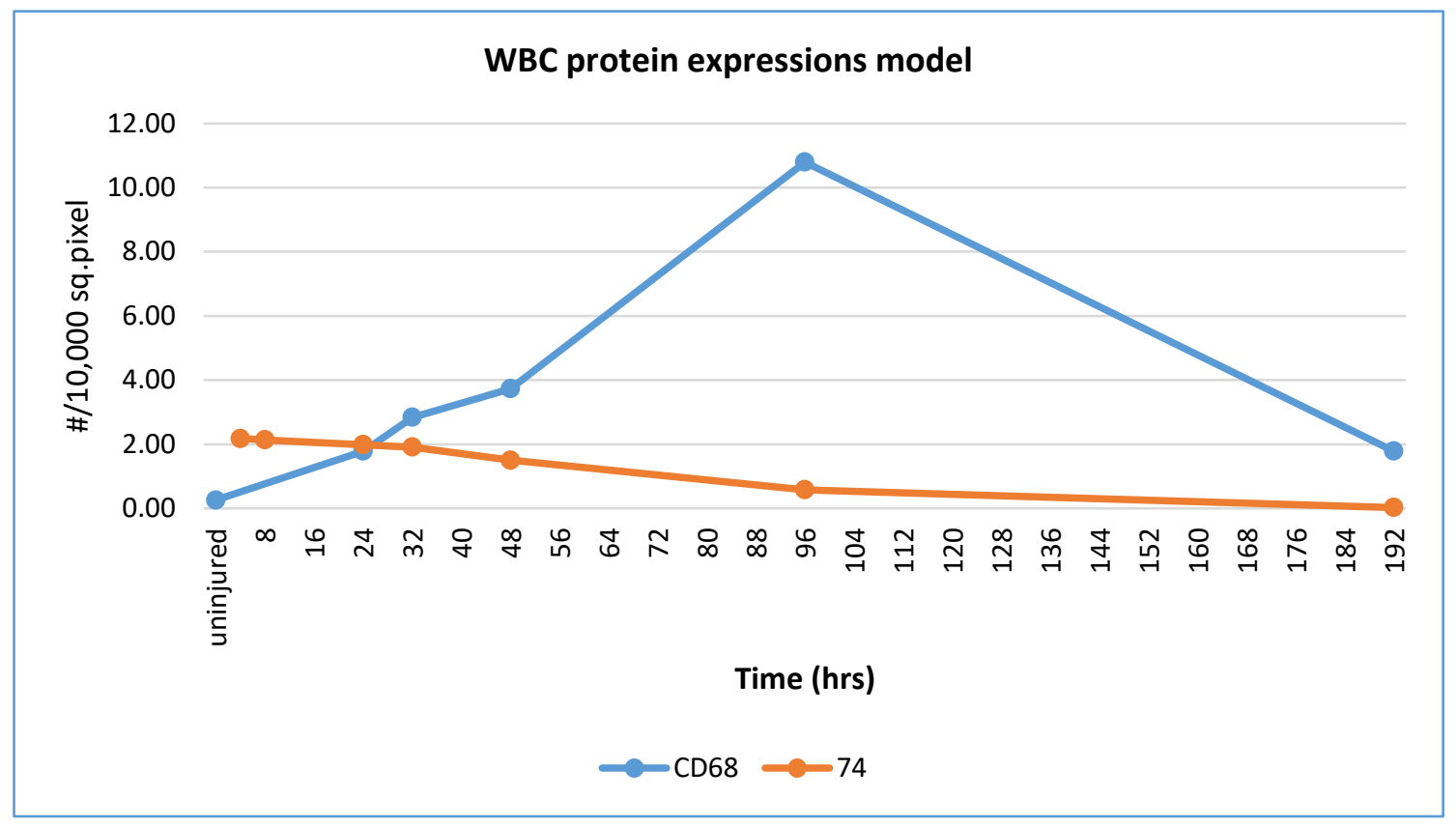

Figure 33 WBC model of CD68 and 7/4 


\section{CHAPTER 8 CONCLUSION}

In this thesis, an automated quantification and analysis framework for muscle healing process is proposed. This framework employs a hybrid image segmentation method which combines the Localized Iterative (LI) Otsu's threshold method assisted by neural networks classifiers and muscle edge detection. Specifically, both LIONN and LIOCNN methods are studied and compared.

For different protein expression of WBCs, the quantified characteristics are analyzed, including the number and size of discrete cells, the number of clustered cells, the total amount of cells, and the density distribution of discrete and clustered cells. Via merging highly accurate classifier results (98.80\% for CD68, 89.30\% for 7/4) with the results generated by the LI Otsu's threshold method, the developed framework automates the processing and analysis of microscopic images and achieves the feature analysis and accuracy that a manual process cannot realize. The experimental results show that LIONN is more suitable for processing images with complicated background (like in 7/4 images) though LIOCNN is better for general cases. Eventually, we have established the timelines for a maximum count of WCB and revealed the model of trend. From the model, it can be seen that the amount of CD68 starts increase after injury and reaches its peak at 96hr. Then it drops.

7/4 decreases from 4-hr after injury. Besides, their traces shows linear.

The following future work will be conducted to advance this research.

$>$ Acquire more training samples for classifiers to improve their accuracy;

Improve the training architecture for NN classifiers;

$>$ Apply the proposed framework to other protein quantification; 
$>$ Study the correlation between different proteins and establish WBC expression model for muscle healing process. 


\section{REFERENCE}

[1] Accession Medical Standards Analysis \& Research Activity, Annual Report 2010, Walter Reed Army Institute of Research, 2010.

[2] Z. Alreza and A. Karimian, "Design a new algorithm to count white blood cells for classification leukemic blood image using machine vision system", in Proc. 6th Int'l Conf. Computer and Knowledge Engineering, Ferdowsi University of Mashhad, 2016, pp. 251-256.

[3] C. Bishop, Neural networks for pattern recognition. New York: OXFORD, 1997, pp. 116-118.

[4] J. Canny, "A computational approach to edge detection", IEEE Trans. Pattern Analysis and Machine Intelligence, vol. PAMI-8, no. 6, pp. 679-698, Nov. 1986.

[5] L. Chua and L. Yang, "Cellular Neural Networks: Applications", IEEE Trans. Circuits and Systems, vol. 35, no. 10, pp. 1273-1290, Oct, 1988.

[6] "Construct Deep Network Using Autoencoders - MATLAB \& Simulink - MathWorks United Kingdom", Mathworks.com, 2017, available at: https://www.mathworks.com/examples/neural-network/mw/nnet-ex33663708-train-stacked-a utoencoders-for-image-classification?s_tid=examples_p1_BOTH.

[7] B. DeKoning, Recruit medicine, 1st ed. Washington, D.C.: Borden Institute, Walter Reed Army Medical Center, 2006, pp. 59-79.

[8] G. Dobek, N. Fulkerson, J. Nicholas and B. Schneider, "Mouse model of muscle crush injury of the legs", Comparative Medicine, pp. 227-232, Jun, 2013.

[9] M. Devi, T. Latha and C. Sulochana, "Iterative thresholding based image segmentation using 2D improved Otsu algorithm", in Proc. Global Conf. Communication Technologies, 2015, pp. 145-149.

[10] Y. Fang, C. Zheng, C. Pan and L. Liu, "White Blood Cell Image Segmentation Using On-line Trained Neural Network", in Proc. IEEE Engineering in Medicine and Biology 27th Annual Conf., 2005, pp. 6476-6479.

[11] C. Ke, "White blood cell detection using a novel fuzzy Morphological shared-weight Neural Network", in Int'l Symp. Computer Science and Computational Technology, Dec. 2008, pp. 532-535.

[12] M. Khan, H. Nisar, N. Aun and P. Lo, "Iterative region based Otsu thresholding of brightfield microscopic images of activated sludg", in Proc. IEEE EMBS Conf. Biomedical Engineering and Sciences, 2016, pp. 533-538.

[13] M. Konstantinovic, P. Lagae, F. Zheng, E. Verbeken, D. De Ridder and J. Deprest, "Comparison of host response to polypropylene and non-cross-linked porcine small intestine serosal-derived collagen implants in a rat model", BJOG: An Int'l J. Obstetrics and Gynaecology, vol. 112, no. 11, pp. 1554-1560, Nov. 2005.

[14] S. Keeling, N. Deashinta, K. Howard, S. Vigil, S. Moonie and B. Schneider, "Macrophage colony stimulating factor-induced macrophage differentiation influences myotube elongation", Biological Research For Nursing, vol. 15, no. 1, pp. 62-70, Jul, 2011. 
[15] S. Khobragade, D. Mor and C. Patil, "Detection of leukemia in microscopic white blood cell images", in Proc. Int'l Conf. Information Processing, 2015, pp. 435-440.

[16] M. Lejeune, J. Jaén, L. Pons, C. López, M. Salvadó, R. Bosch, M. García, P. Escrivà, J. Baucells, X. Cugat and T. Álvaro, "Quantification of diverse subcellular immunohistochemical markers with clinicobiological relevancies: validation of a new computer-assisted image analysis procedure", Journal of Anatomy, vol. 212, no. 6, pp. 868-878, Jun, 2008.

[17] Y. LeCun, B. Boser, J. Denker, D. Henderson, R. Howard, W. Hubbard and L. Jackel, "Backpropagation Applied to Handwritten Zip Code Recognition", Neural Computation, vol. 1, no. 4, pp. 541-551, winter 1989.

[18] Y. Lecun, L. Bottou, Y. Bengio and P. Haffner, "Gradient-based learning applied to document recognition", Proc. of the IEEE, vol. 86, no. 11, pp. 2278-2324, 1998.

[19] J. Molloy, D. Feltwell, S. Scott and D. Niebuhr, "Physical training injuries and interventions for military recruits", Military Medicine, vol. 177, no. 5, pp. 553-558, May 2012.

[20] S. Manik, L. Saini and N. Vadera, "Counting and Classification of White Blood Cell using Artificial Neural Network (ANN)", in Proc. 1st IEEE Inter'l Conf. Power Electronics. Intelligent Control and Energy Systems, 2017 pp. 1-5.

[21] D. Niebuhr, C. Scott, T. Powers, Y. Li, W. Han, A. Millikan and M. Krauss, "Assessment of recruit motivation and strength study: preaccession physical fitness assessment predicts early attrition", Military Medicine, vol. 173, no. 6, pp. 555-562, Jun, 2008.

[22] V. Nair and G. E. Hinton. Rectified linear units improve restricted Boltzmann machines. In Proc. 27th Int'l Conf. Machine Learning, 2010 pp. 807-814.

[23] N. Otsu, "A threshold selection method from gray-level histograms", IEEE Trans. Systems, Man, and Cybernetics, vol. 9, no. 1, pp. 62-66, Jan. 1979.

[24] "Object Detection Using Deep Learning - MATLAB \& Simulink Example - MathWorks United Kingdom", Mathworks.com, 2017, available at: https://www.mathworks.com/help/vision/examples/object-detection-using-deep-learning.html

[25] C. Peixinho, L. Oliveira and J. Machado, "Following-up the regeneration of injured rat muscle through the average pixel intensity of ultrasound biomicroscopic images", in Proc. Joint UFFC, EFTF and PFM Symp., 2013, pp. 888-890.

[26] J. Pan, X. Zheng, L. Sun, L. Yang, Y. Wang, H. Lou and P. Wang, "Image segmentation based on 2D Otsu and simplified swarm optimization", in Proc. Int'l Conf. Machine Learning and Cybernetics, 2016, pp. 1026-1030.

[27] J. Prinyakupt and C. Pluempitiwiriyawej, "Segmentation of white blood cells and comparison of cell morphology by linear and naïve Bayes classifiers", BioMedical Engineering OnLine, vol. 14, no. 1, Jun, 2015.

[28] B. Schneider, S. Moonie and S. Vigil, "The Relation Between Immunohistochemical Product Discreteness and Leukocyte Manual Quantification Reliability", Applied Immunohistochemistry \& Molecular Morphology, vol. 18, no. 6, pp. 555-560, Feb, 2010. 
[29] C. Sha, J. Hou, H. Cui and J. Kang, "Gray level-median histogram based 2D Otsu's method", in Proc. Int'l Conf. Industrial Informatics-Computing Technology, Intelligent Technology, Industrial Information Integration, 2015, pp. 30-33.

[30] C. Spadaccio, A. Rainer, S. Porcellinis, F. Marco, M. Chello, M. Trombetta and J. Genovese, "Muscle reconstruction and regeneration using biodegradable scaffolds", in Proc.Advanced Technologies for Enhancing Quality of Life, 2010, pp. 104-109,.

[31] F. Sadeghian, Z. Seman, A. Ramli, B. Abdul Kahar and M. Saripan, "A framework for white blood cell segmentation in microscopic blood images using digital image processing", Biological Procedures Online, vol. 11, no. 1, pp. 196-206, Jun, 2009.

[32] H. Sun, Y. Ma, H. Wu, L. Miao, X. Chen, X. Ji and P. Cai, "An improved OTSU's method for CT image boundary contour extraction", in Proc. IEEE Int'l Conf. Imaging Systems and Techniques (IST), 2016, pp. 493-497.

[33] J. Sa, X. Sun, T. Zhang, H. Li and H. Zeng, "Improved Otsu segmentation based on Sobel operator", in Proc. 3rd Int'l Conf. Systems and Informatics, 2016, pp. 886-890.

[34] N. Salem, "K4. segmentation of white blood cells from microscopic images using K-means clustering", in Proc. 31st National Radio Science Conf., 2014, pp. 371-376.

[35] W. Shitong and W. Min, "A New Detection Algorithm (NDA) Based on Fuzzy Cellular Neural Networks for White Blood Cell Detection", IEEE Trans. Information Technology in Biomedicine, vol. 10, no. 1, pp. 5-10, Jan. 2006.

[36] H. Talebi, A. Davoudi, A. Mohammadi, M. Menhaj, A. Khoshdel and M. Ghorbani, "Automatic recognition of white blood cells using weighted two phase test sample sparse representation", in Proc. 6th Int'l Conf. Computer and Knowledge Engineering, 2016, pp. 25-29.

[37] N. Theera-Umpon and P. Gader, "System-level training of neural networks for counting white blood cells", IEEE Trans. Systems, Man and Cybernetics, Part C (Applications and Reviews), vol. 32, no. 1, pp. 48-53, Feb. 2002.

[38] Technical Bulletin Medical 592. U.S. Department of the Army, "Prevention and control of musculoskeletal injuries associated with physical training", Department of the Army, Washington, DC, 2011. 
Date: July 2017

\section{CURRICULUM VITAE}

\section{Yang Jiao}

4505 S Maryland Pkwy, Las Vegas, NV

Department of Electrical and Computer Engineering

University of Nevada, Las Vegas

Cell phone: 702-609-3573

Email: jiaoy1@unlv.nevada.edu

Education

Queen Mary, University of London

Bachelor, Engineering, June 2015

Beijing University of Telecommunications and Posts

Bachelor, Engineering, June 2015 